\title{
Rigorous embedding of cell dynamics simulations in the Cahn-Hilliard-Cook framework: Imposing stability and isotropy
}

\author{
G. J. A. Sevink* \\ Leiden Institute of Chemistry, Leiden University, P. O. Box 9502, 2300 RA Leiden, The Netherlands \\ (Received 27 January 2015; revised manuscript received 15 April 2015; published 29 May 2015)
}

\begin{abstract}
We have rigorously analyzed the stability of the efficient cell dynamics simulations (CDS) method by making use of the special properties of the local averaging operator $\langle\langle *\rangle\rangle-*$ in matrix form. Besides resolving a theoretical issue that has puzzled many over the past three decades, this analysis has considerable practical value: It relates CDS directly to finite-difference approximations of the Cahn-Hilliard-Cook equations and provides a straightforward recipe for replacing the original two- or three-dimensional (2D or 3D) averaging operators in CDS by an equivalent (in terms of stability) discrete Laplacian with superior isotropy and scaling behavior. As such, we open up a route to suppress the unphysical reflection of the computational grid in CDS results (grid artifacts). We found that proper rescaling of discrete Laplacians, needed to employ them in CDS, is equivalent to introducing a well-chosen time step in CDS. In turn, our analysis provides stability conditions for phase-field simulations based on the Cahn-Hilliard-Cook equations. Subsequently, we have quantitatively compared the isotropy and scaling behavior of several discrete 2D or 3D Laplacians, thereby extending the significance of this work to general field-based methodology. We found that all considered discrete Laplacians have equivalent scaling behavior along the Cartesian directions. In addition, and somewhat surprisingly, known "isotropic" discrete Laplacians, i.e., isotropic up to fourth order in $|\mathbf{k}|$, become quite anisotropic for larger wave vectors, whereas "less isotropic" discrete Laplacians (second order) are only slightly anisotropic on the whole $|\mathbf{k}|$ range. We identified a hard limit to the accuracy with which the discrete Laplacian can emulate the two important properties of the optimal (continuum) Laplacian, as an improvement of the isotropy, by introducing additional points to the stencil, will negatively affect the scaling behavior. Within this limitation, the discrete compact Laplacians in the Dn Qm class known from lattice hydrodynamics, $D 2 Q 9$ in $2 \mathrm{D}$ and $D 3 Q 19$ in $3 \mathrm{D}$, are found to be optimal in terms of isotropy. However, by being only slightly anisotropic on the whole range and enabling larger time steps, the discrete Laplacians that relate to the local averaging operator of Oono and Puri (2D) and Shinozaki and Oono (3D) as well as the less familiar 3D discrete $B v V$ Laplacian developed for dynamic density functional theory are valid alternatives.
\end{abstract}

DOI: 10.1103/PhysRevE.91.053309

PACS number(s): 02.60.Lj, 64.75.Jk

\section{INTRODUCTION}

The phenomenon of spinodal decomposition in systems with only one scalar order parameter, i.e., binary mixtures, like metal alloys and polymer blends, or diblock copolymers, has been intensively studied by experiment, theory, and simulation. One may therefore be tempted to conclude that the technologically relevant fundamental information on both the kinetics and the resulting morphologies is sufficiently known. Although this is certainly partially true, the study of the growth of order through domain coarsening (or phase ordering dynamics) [1] remains challenged by a number of issues, two of which we discuss in more detail. First, phase ordering in experiments is often a process of hours or days and it is uncertain if true equilibrium is ever reached, since the driving forces for coarsening are usually minute. An illustrative example of such "slow" dynamics is the annihilation of topological defects in diblock copolymers, with a defect density that decays only algebraically as $t^{v}$, with $t$ the experimental time and $v$ a small negative factor depending on structure connectivity [2]. While theoretical study of such processes relies on a "simple" model and often concentrates on limiting (at or near equilibrium) behavior [1], computational methods can provide direct insight in detailed dynamic properties and

\footnotetext{
*a.sevink@chem.leidenuniv.nl
}

nonequilibrium morphologies. Nevertheless, these approaches should be able to simulate, in addition to trajectories long enough for capturing slow phenomena, large volumes to ensure proper statistics and minimize the role of (periodic) boundary conditions, ruling out most methodologies that are directed to the microscopic time and length scales. As a result, there is still a considerable computational challenge in directly relating experimental emergent behavior to simulation results, in particular if one is also interested in including or elucidating more detailed (molecular) information. Second, spinodal decomposition often competes with several internal-impurities (in alloys) [3] and symmetry breaking by confinement in thin films [4] and/or reactions if present [5] —and external factors, including fields that are applied to accelerate defect demoval and tailor the overall structure and/or structure orientation (see, for instance, Ref. [6]). These factors pose new theoretical challenges, as they should be appropriately accounted for in a model description, and provide new insights. We therefore conclude that computational study of such systems is still of considerable importance.

The standard model for phase ordering kinetics on a coarsegrained continuum level is the time-dependent Ginzburg Landau (TDGL, nonconserved) or Cahn-Hilliard-Cook (CHC, conserved) equation, which provides a model for the diffusion of a scalar order parameter field $\psi(\mathbf{r}, t)$ given an appropriate free-energy functional $F[\psi]$, which implicitly acknowledges the underlying molecular or microscopic detail [1]. 
Computational studies based on finite-difference approximations of these partial differential equations (PDEs) have elucidated many aspects of phase separation for a whole range of systems [7]. In 1987, Oono and Puri proposed an alternative approach, a cellular automaton called cell dynamics simulations (CDS) [8], driven by the conviction that modeling spinodal decomposition by PDEs is not more fundamental than discrete modeling [9]. Although the initial idea was to grasp a deeper mathematical meaning of phase ordering by disregarding a microscopic reality, a more practical goal was to open up an efficient route to include hydrodynamic interactions in a description of spinodal decomposition. Although this promise was never fulfilled, CDS is considerably more efficient than discretized TDGL-CHC in describing the phase separation dynamics in many "soft" systems, which effectively undergo purely diffusive dynamics. Starting from a scalar order parameter $\psi, \mathrm{CDS}$ relies on a one-to-one map $f: \mathbb{R} \rightarrow \mathbb{R}$ with two hyperbolic sinks and one hyperbolic source and discrete dynamics,

$$
\begin{aligned}
\psi(\mathbf{n}, t+1)= & \mathcal{F}[\psi(\mathbf{n}, t)]=f[\psi(\mathbf{n}, t)] \\
& +D[\langle\psi(\mathbf{n}, t)\rangle\rangle-\psi(\mathbf{n}, t)],
\end{aligned}
$$

where the first term represents the flow due to the map and the second term, with $\langle\langle *\rangle\rangle$ an isotropic local average over the neighborhood except for the center cell $\mathbf{n}$, introduces the intercell coupling with $D$ a rate constant [10]. The choice of the latter was based on the idea that capturing the spherical mean value theorem for harmonic functions was the right way for averaging [9]. Since the net gain of the order parameter by the center cell $\mathbf{n}$ is $\mathcal{F}[\psi]-\psi$, the discrete model for the conserved case reads

$$
\psi(\mathbf{n}, t+1)=\mathcal{F}[\psi(\mathbf{n}, t)]-\langle\langle\mathcal{F}[\psi(\mathbf{n}, t)]-\psi(\mathbf{n}, t)\rangle .
$$

As discussed in detail by Oono and Puri [8], the map only has to satisfy the previously mentioned properties and does not necessarily have to relate to an underlying free-energy functional. Several maps were tried, without much effect on the simulation results, after which they selected a simple $f(\psi)=$ $A \tanh (\psi)$ map, where $A(A=1.3$ [8] and $A=1.5$ [11]) can be matched for particular systems. The CDS methodology has been applied to study the diffusive structure evolution in binary blends of both polymers and alloys [5,8,10-12] and diblock copolymers [13] as well as reaction-diffusion systems of the Fischer type for studying chemical reactions and population dynamics [14]. More recently, CDS has gained popularity as an efficient model for studying phase separation in diblock copolymers that is modulated by additional factors, such as confinement, shear, and electric fields, see the recent review in Ref. [6] and references therein. A favorable comparison between a molecular field theory and CDS, for a lamellaforming diblock copolymer melt under an electric field, was also recently published [15].

Despite the conceptually different roots, CDS is usually discussed in close relation to the TDGL-CHC equations, starting from the earliest publications [10]. Because the differences are quite subtle, many later authors have simply presented CDS as an efficient finite-difference approximation of these PDEs. Nevertheless, a rigorous derivation of such a relation, in the spirit of those that relate lattice Boltzmann to a finite-difference approximation of the Navier-Stokes equation [16,17], is lacking. Moreover, studies that assume this relation have failed to explain the remarkable stability of CDS for large (effective) time steps [18]. This is somewhat worrisome, as it is particularly the large time step that renders CDS most efficient for studying spinodal decomposition [19]. Consequently, the CDS methodology has come to somewhat of a standstill in terms of further development, since it cannot benefit from improved finite-difference Laplacians (in short: stencils) that have been developed more recently in other areas of computational research. Here, we analyze and proof the stability of (conserved) CDS. Moreover, we highlight the actual role of the original $\langle\langle *\rangle\rangle-*$ operation in CDS and show how it can be replaced by a finite-difference Laplacian, thereby embedding CDS in the CHC framework and enabling an inspired choice of the stencil and the associated stable time step. In the second part of the paper, we evaluate the properties of a number of often-used stencils in detail, focusing on the scaling behavior and isotropy. For completeness, we start with a historic overview of the CDS development and illustrate the background of currently unresolved issues.

\section{A. Relating CDS to time-dependent Ginzburg Landau?}

After introducing the CDS method in a short paper in Physical Review Letters [8], the same authors published a separate paper containing a more detailed analysis of CDS [10]. There they directly related the Euler-discretized deterministic TDGL equation, based on a Ginzburg-Landau-Wilson $\phi^{4}$ free energy for critical systems, given by [10]

$$
\begin{aligned}
\psi(\mathbf{n}, t+1)= & \bar{A} \psi(\mathbf{n}, t)-(\bar{A}-1) \psi(\mathbf{n}, t)^{3} \\
& +D[\langle\psi(\mathbf{n}, t)\rangle\rangle-\psi(\mathbf{n}, t)],
\end{aligned}
$$

to the discretized equation obtained from the deterministic TDGL equation using a semigroup map,

$$
\begin{aligned}
\psi(\mathbf{n}, t+1)= & \frac{A(\psi(\mathbf{n}, t))}{\left[1+\psi(\mathbf{n}, t)^{2}\left(A^{2}-1\right)\right]^{\frac{1}{2}}} \\
& +D[\langle\psi(\mathbf{n}, t)\rangle\rangle-\psi(\mathbf{n}, t)] .
\end{aligned}
$$

For completeness, we note that both expressions were obtained by rescaling, i.e., $2 L \tau \Delta t \rightarrow \Delta t, \sqrt{g / \tau} \psi \rightarrow \psi$, and $\Delta t /\left(\tau \Delta x^{2}\right) \rightarrow D$, with $g$ and $\tau$ positive constants in the free energy and $\bar{A}=1+\Delta t / 2$ (for details, see Ref. [10]). For clarity, we have used the original notation, but in the remainder we feel free to reuse symbols. One finds that (4) is the CDS equation for the nonconserved order parameter (1), since differences between the map $f(\psi)=A \tanh (\psi)$ and the first term in (4) are irrelevant. For small $\psi$, both expressions can be matched by setting $\bar{A}=A$, providing $\Delta t=0.6(A=1.3)$ and $\Delta t=1.0(A=1.5)$, respectively. From the observation that these time increments are rather extreme for a Euler scheme, Oono and Puri concluded that CDS should not be justified in terms of a (Euler) discretized deterministic TDGL equation. The conserved case can be obtained after application of the discrete Laplacian $\langle\langle *\rangle\rangle-*$ to (4), where they note that this operation should be interpreted as mere local averaging [10]. Numerical simulations were employed to empirically determine a proper range of $A$ and $D$ values (see Fig. 21 in 
Oono and Puri), and unphysical checkerboard patterns were found in a small region cramped between $F$, i.e., patterns that freeze into metastable states, and $\infty$, where there is an arithmetic overflow. Based on a comparison of results of (linear) stability analysis and these numerical results, the nonlinear term was found to have a stabilizing effect.

\section{B. Linear stability analysis using TDGL}

Later contributions disregarded these early remarks of Oono and Puri. Teixeira and Mulder, for instance, exploited the apparent relation between CDS and the Euler-discretized deterministic TDGL-CHC equations, based on the recognition that the CDS method has essentially the same dynamical scaling properties as the $\mathrm{CHC}$ equation [18]. They concentrated on the properties of the operator $\langle\langle *\rangle\rangle-*$ for local averaging in an attempt to explain the puzzling stability of CDS. Defining $\psi(\mathbf{n}, t+1)-\psi(\mathbf{n}, t)$ as a discrete representation of $\partial \psi / \partial t$ and $\langle\langle *\rangle\rangle-*$ as the discrete Laplacian $\nabla^{2} *$, one can write the discrete CDS equation (2) in the dimensionless form of the deterministic Cahn-Hilliard-Cook equation [20,21],

$$
\begin{aligned}
\frac{\partial \psi}{\partial t} & =\nabla^{2}\left[-(A-1) \psi+\frac{A}{3} \psi^{3}-D \nabla^{2} \psi\right] \\
& =\frac{1}{2} \nabla^{2}\left(-\psi+\psi^{3}-\nabla^{2} \psi\right)
\end{aligned}
$$

after expanding $f(\psi)=A \tanh (\psi) \approx A \psi-A / 3 \psi^{3}$ and taking the usual values $D=1 / 2$ and $A=3 / 2$ (note the condition $A>1)$. Linear stability analysis can be used to determine stable time increments by considering the linearized equation (5) about the homogeneous fixed point, i.e., $\psi(\mathbf{n})=\psi_{0}$ or $\psi_{\mathbf{k}}=\psi_{0} \delta_{\mathbf{k}, 0}$ in Fourier space, corresponding to the single phase state of the system [20]. With the discrete Laplacian $L(\mathbf{k})$ yet unspecified, Teixeira and Mulder obtain in the Fourier domain (using $\partial \psi / \partial t=[\psi(t+1)-\psi(t)] / \Delta t$ instead) [18]

$$
\begin{aligned}
\psi_{\mathbf{k}}(t+\Delta t) & =\left[1-(A-1) \Delta t L(\mathbf{k})-D \Delta t L^{2}(\mathbf{k})\right] \psi_{\mathbf{k}}(t) \\
& =H_{\mathbf{k}} \psi_{\mathbf{k}}(t)
\end{aligned}
$$

meaning that, for the critical quench $\left(\psi_{0}=0\right)$, a small perturbation $\delta \psi_{\mathbf{k}}$ is amplified by a factor $H_{\mathbf{k}}$. Linearizing the original deterministic TDGL [see (3)] will provide the same expression but for a restricted set of values for $A, D$, and $\Delta x$. For $H_{\mathbf{k}}>1$, i.e., $-L(\mathbf{k})<(A-1) / D$, tangential bifuraction leads to the growth of small $\mathbf{k}$ modes, while for $H_{\mathbf{k}}<-1$, or

$$
H_{\mathbf{k}}^{\prime}=-(A-1) \Delta t L(\mathbf{k})-D \Delta t L^{2}(\mathbf{k})<-2,
$$

subharmonic bifurcations lead to the growth of large-k modes. One should note that $H_{\mathbf{k}}^{\prime}$ is quadratic in $L(\mathbf{k})$; moreover, since $L(\mathbf{0})=0$ and $L(\mathbf{k}) \sim-\mathbf{k}^{2} \leqslant 0 \forall \mathbf{k}$, it is easy to see that $H_{\mathbf{k}}^{\prime}>$ $-2 \forall \mathbf{k}$ if $H_{\mathbf{k}}^{\prime}>-2$ for a $\mathbf{k}$ vector for which $L(\mathbf{k})$ is minimal. Teixeira and Mulder considered several discrete Laplacians $L(\mathbf{k})$ and found the largest time increment,

$$
\Delta t<\frac{\Delta x^{4}}{18 D-3(A-1) \Delta x^{2}},
$$

for the original discrete Laplacian of Oono and Puri [further denoted by $L(\mathbf{k})_{\mathrm{OPs}}$ ] [8] or, for the usual values of $A=3 / 2$, $D=1 / 2$ and $\Delta x=1, \Delta t<0.12$. In terms of isotropy, $L(\mathbf{k})_{\text {OPs }}$ was shown only to be isotropic up to second order in $k$ (from an expansion around $\mathbf{k}=\mathbf{0}$ ), but Teixeira and Mulder concluded that the use of the Oono-Puri Laplacian is desired because it leads to the least severe violation of the stability conditions. Clearly, for the (implicit) choice $\Delta t=1$, the conclusion should be that the CDS scheme is unstable for $L(\mathbf{k})_{\mathrm{OPs}}$ and all other discrete two-dimensional (2D) Laplacians considered by Teixeira and Mulder [18].

\section{DETERMINING STABILITY VIA AN ALTERNATIVE APPROACH}

An alternative conclusion, in line with the observation of Oono and Puri and the numerically confirmed stability of CDS [10], is that this analysis is simply not applicable for the CDS method. Moreover, we note that the maximum time increment derived by this analysis does not provide much useful information about the quality of the discrete Laplacian in terms of isotropy and scaling behavior. If we assume that a "perfect" isotropic discrete 2D Laplacian $L_{p}(\mathbf{n})$ exists on a grid of dimensions $N_{x} \times N_{y}$, i.e., $L_{p}(\mathbf{k})=-\mathbf{k}^{2}$ for $k_{i} \in\left\{2 \pi / N_{i}, \ldots, \pi\right\}$ (all in units $1 / \Delta x$ ), we obtain from $\min _{\mathbf{k}} L_{p}(\mathbf{k})=-\pi^{2} / \Delta x^{2}$ and (7) (for the usual values of $A$, $D$, and $\Delta x$ )

$$
\Delta t_{p}<\frac{4}{\pi^{4}-\pi^{2}} \approx 0.05,
$$

resulting in classification as a less "convenient" discrete Laplacian, despite the fact that it provides a superior physical description. To better understand stability, it makes sense to rewrite the CDS equations in a matrix-vector notation [after expanding $f(\psi)$ ],

$$
\vec{\psi}_{t+1}=\vec{\psi}_{t}+L\left[(1-A) \vec{\psi}_{t}+\frac{A}{3} \Phi^{2} \vec{\psi}_{t}-D L \vec{\psi}_{t}\right]
$$

with $n=N_{x} N_{y} N_{z}$ the dimension of the state space, $\vec{\psi}=$ $\left(\psi_{0}, \ldots, \psi_{n}\right) \in \mathbb{R}^{n}$ and $\Phi, L \in \mathbb{R}^{n \times n}$ are $n \times n$ matrices. To avoid confusion, we note that the order parameter $\psi$ remains scalar and that (10) is just an alternative way of writing the expression (2) for all cells. The matrix $\Phi$ is diagonal with $\Phi_{i i}=$ $\psi_{i}$ and $L$ represents the discrete Laplacian in the terminology of Teixeira and Mulder, although it actually represents the averaging operator. Moreover, $\Phi$ and $L$ do not commute. We may assume that the matrix $L$ is real and symmetric, meaning that $D=Q^{T} L Q$ is a diagonal matrix containing eigenvalues and $Q$ is a unitary matrix containing the eigenvectors (as columns). We can therefore use the $n$ eigenvectors or states $\vec{\psi}^{j}(j=1, \ldots, n)$, i.e., with $L \vec{\psi}^{j}=\lambda_{j} \vec{\psi}^{j}$, as a basis for $\mathbb{R}^{n}$. Writing $\vec{\psi}_{t}=\sum_{j=1}^{n} a_{j} \vec{\psi}^{j}$ and $\vec{\psi}_{t}^{\prime}=\Phi^{2} \vec{\psi}_{t}=\sum_{j=1}^{n} b_{j} \vec{\psi}^{j}$,

$$
\begin{aligned}
\vec{\psi}_{t+1} & =\sum_{j=1}^{n} a_{j}\left[1+\lambda_{j}\left(1-A+\frac{A}{3} \frac{b_{j}}{a_{j}}\right)-\lambda_{j}^{2} D\right] \vec{\psi}^{j} \\
& =\sum_{j=1}^{n} a_{j} p_{j} \vec{\psi}^{j},
\end{aligned}
$$

with $p_{j}$ the multiplication factor, which depends on $\lambda_{j}$. Without the nonlinear term in (10), the iterative scheme is stable for

$$
\left|1+\lambda_{j}(1-A)-\lambda_{j}^{2} D\right|=\left|p_{j}^{l}\right| \leqslant 1 \quad \forall j,
$$


with $p_{j}^{l}$ the multiplication factor for the linearized case Moreover, since $A>1$ and $b_{j} / a_{j}>0$, it is indeed clear that the nonlinear term stabilizes the iterative scheme [10]. The two-dimensional discrete Laplacian of Oono and Puri [8] reads (without a $1 / \Delta x^{2}$ prefactor [10], which is equal to setting $\Delta x=1)$

$$
\begin{aligned}
\langle\langle f(\mathbf{n}, t)\rangle\rangle-f(\mathbf{n}, t) & =\frac{1}{6} \sum_{i=1}^{4} f_{i}^{(1)}+\frac{1}{12} \sum_{i=1}^{4} f_{i}^{(2)}-f^{(0)} \\
& =L(\mathbf{n})_{\mathrm{OP}},
\end{aligned}
$$

where we used the shorthand notation of Thampi et al. [22], i.e., $f_{i}^{(j)}=f\left(\mathbf{n}+\mathbf{n}_{i}^{j}\right)$, with $\mathbf{n}_{i}^{j}$ a class of $N_{j}$ vectors of the same length (in units of the discretization length $\Delta x$ ) in the first shell surrounding $\mathbf{n}$. In the CDS literature, these points are known as nearest neighbors $(j=1)$ and next-nearest neighbors $(j=2)$. For a cubic grid, they are $N_{0}=1$ null vector with $\left\|\mathbf{n}^{0}\right\|=0$, $N_{1}=4$ vectors of length $\left\|\mathbf{n}_{i}^{1}\right\|=1$ and $N_{2}=4$ vectors of length $\left\|\mathbf{n}_{i}^{2}\right\|=\sqrt{2}$.

Filling the matrix with the weights given in (13) and accounting for periodic boundary conditions, where we have considered $N_{x}=N_{y}$ up to $N_{x}=50$, including the $40 \times 40$ lattice considered for numerical analysis [10], indeed provides a symmetric matrix $L$. Determination of the eigenvectors and eigenvalues, by a Jacobi method, provides an orthonormal basis of eigenvectors $\vec{\psi}^{j}$. We find $-1.333 \leqslant \lambda_{j} \leqslant 0 \forall j$ for all considered lattice sizes (see Fig. 1). From condition (12) for the linearized case, it is easy to define two domains for the eigenvalues:

$$
\begin{gathered}
{\left[(1-A)-\sqrt{(1-A)^{2}+8 D}\right] / 2 D \leqslant \lambda_{j} \leqslant(1-A) / D} \\
0 \leqslant \lambda_{j} \leqslant\left[(1-A)+\sqrt{(1-A)^{2}+8 D}\right] / 2 D,
\end{gathered}
$$

in which the CDS scheme is stable, i.e., the scheme does not result in an arithmetic overflow. For the usual values $(A, D)=$ $(3 / 2,1 / 2)$, these domains are given by $(-1-\sqrt{17}) / 2 \approx$ $-2.56 \leqslant \lambda_{j} \leqslant-1$ or $0 \leqslant \lambda_{j} \leqslant(-1+\sqrt{17}) / 2 \approx 1.56$. This

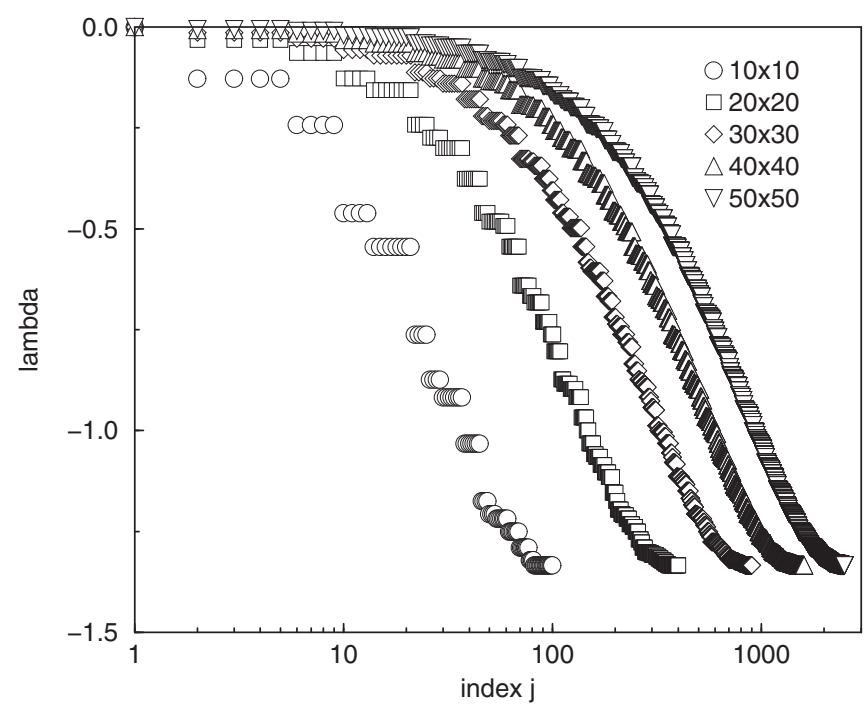

FIG. 1. Numerically determined values of the eigenvalue $\lambda_{j}$ of the averaging operator $L$ of Oono and Puri for an $N \times N$ lattice $(N=$ $10,20,30,40,50)$. suggests that coefficients corresponding to eigenvalues $\lambda_{j}$ of $L$ in the range $\lambda_{j} \in(-1,0)$ scale by a factor $p_{j}^{l}>1$ at every time step (note that $p_{j}^{l} \leqslant 1.125$ ). As a result, they will diverge for $t \rightarrow \infty$.

However, one should note that the analysis is based on the map $f(\psi)=A \psi$ and that the free energy associated with this map carries minima at $\pm \infty$. For the actual map $f(\psi)=$ $A \psi-(A / 3) \psi^{3}$, we may write $\vec{\psi}_{t}$ as $\vec{\psi}_{t}=\vec{\psi}_{t}-\vec{\psi}_{t}^{r}+\vec{\psi}_{t}^{r}$, with $\vec{\psi}_{t}^{r}=\sum_{j=1}^{n} c_{j} \vec{\psi}^{j}$ a reference state $\left[\left(\vec{\psi}_{t}^{r}\right)^{\prime}=\sum_{j=1}^{n} d_{j} \vec{\psi}^{j}\right]$ for which the entries $\psi_{i}$ satisfy $\psi_{i}=f\left(\psi_{i}\right)=A \psi_{i}-(A / 3) \psi_{i}^{3}$. From (10), we can write the update as

$$
\begin{aligned}
\vec{\psi}_{t+1} & =\sum_{j=1}^{n}\left[\left(a_{j}-c_{j}\right) p_{j}+c_{j} p_{j}^{\prime}\right] \vec{\psi}^{j} \\
& =\vec{\psi}_{t+1}^{r}+\sum_{j=1}^{n}\left(a_{j}-c_{j}\right) p_{j} \vec{\psi}^{j}
\end{aligned}
$$

with $p_{j}^{\prime}=\left(1-D \lambda_{j}^{2}\right)$, i.e., $\left|p_{j}^{\prime}\right|<1$ for all eigenvalues $\lambda_{j}$ of $L$ for $D \leqslant 9 / 8\left(\left|p_{j}^{\prime}\right|=1\right.$ only for $\left.\lambda=0\right)$, and

$$
p_{j}=1+\lambda_{j}\left(1-A+\frac{A}{3} \frac{b_{j}-d_{j}}{a_{j}-c_{j}}\right)-\lambda_{j}^{2} D .
$$

From $\psi_{i}=f\left(\psi_{i}\right)$, we find that $\psi_{i}=0$ or $\psi_{i}=$ $\pm \sqrt{(3 A-3) / A}$. Setting $\psi_{i}=\psi_{i}^{r}+\delta_{i}$, we find $\psi_{i}^{3} \approx\left(\psi_{i}^{r}\right)^{3}+$ $3 \delta_{i}\left(\psi_{i}^{r}\right)^{2}$ when $\delta_{i}$ is small. Defining $\vec{\delta}=\left(\delta_{0}, \ldots, \delta_{n}\right) \in \mathbb{R}^{n}$ and inserting the relevant values for $\psi_{i}^{r}$, we thus find that $\vec{\psi}_{t}^{\prime}=$ $\left(\vec{\psi}_{t}^{r}\right)^{\prime}+(9-9 / A) \vec{\delta}$ or, with $\vec{\delta}=\sum_{j=1}^{n} \epsilon_{j} \vec{\psi}^{j}, a_{j}=c_{j}+\epsilon_{j}$ and $b_{j}=d_{j}+\epsilon_{j}(9-9 / A)$. Substitution in (16) provides

$$
p_{j}=1+\lambda_{j}(2 A-2)-\lambda_{j}^{2} D,
$$

and since $A>1, p_{j}<1$ for all eigenvalues of $L$ except for $\lambda_{j}=0\left(p_{j}=1\right)$. Moreover, the perturbation is not amplified if $p_{j} \geqslant-1$, leading to the condition

$$
D \leqslant \frac{3}{8}(7-4 A) \text {. }
$$

We thus conclude that the CDS scheme that uses the map $f(\psi)=A \psi-(A / 3) \psi^{3}$ is stable provided that (18) holds. Figure 2 shows a direct comparison of the analytical prediction (18) for the boundary of the "safe" region and numerical results for a large lattice and many steps. The excellent agreement between analytic and numerical results shows that our analytical analysis works very well and explains why CDS is stable. Clearly, since CDS for the original map $f(\psi)=A \tanh (\psi)$ was numerically found to be stable for $D=1 / 2$ and $A=3 / 2$, the "safe" region for the original map is slightly larger. We can understand this intuitively in terms of a smaller derivative but will not perform the analysis here. We note that the empirically determined "safe" regions in the $A-D$ plane, see Fig. 21 in Oono and Puri [10], are for a nonconserved model (linear in $\lambda_{j}$ ) and for an alternative model.

\section{A. Averaging and the discrete Laplacian}

In the previous subsections, we have referred to the $\langle\langle *\rangle\rangle-*$ operator as the discrete Laplacian. Oono and Puri [10] explicitly noted that this operation facilitates local averaging rather than an operation that comes from the Laplacian for the 


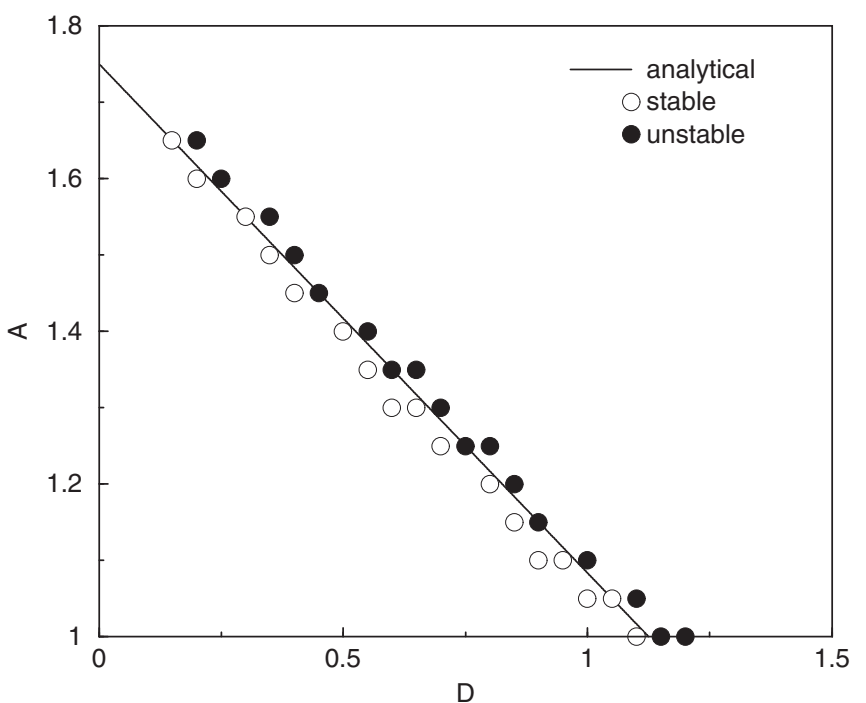

FIG. 2. Comparison of the analytically as determined boundary of the "safe" region [CDS is stable for combinations $(A, D)$ left of the line] and stability determined by simulation [50000 steps of CDS, $100 \times 100$ lattice]. Both models are based on $f(\psi)=A \psi-(A / 3) \psi^{3}$ and the averaging operator $L$ of Oono and Puri. Close to the line, there were a few cases where we did not encounter arithmetic overflow but instead a clear checkerboard pattern was visible at the end of simulation. Since the number of steps is finite, we classified such results as unstable.

continuum field. Using the results of the last subsection, we can understand this reasoning. If we derive a discretized 2D Laplacian similar to the one used by Oono and Puri [10], we have to rescale the weights, i.e.,

$$
\begin{aligned}
\langle\langle f(\mathbf{n}, t)\rangle\rangle-f(\mathbf{n}, t) & =\frac{1}{2}\left[\sum_{i=1}^{4} f_{i}^{(1)}+\frac{1}{2} \sum_{i=1}^{4} f_{i}^{(2)}-6 f^{(0)}\right] \\
& =L(\mathbf{n})_{\mathrm{OPs}},
\end{aligned}
$$

to obtain proper $-\mathbf{k}^{2}$ scaling in Fourier space. The importance of this rescaling was already mentioned by Tomita [23], who introduced a family of properly scaled discrete operators representing the Laplacian as

$$
L(\mathbf{n})_{\epsilon}=\frac{1}{1+2 \epsilon}\left[\sum_{i=1}^{4} f_{i}^{(1)}+\epsilon \sum_{i=1}^{4} f_{i}^{(2)}-4(1+\epsilon) f^{(0)}\right]
$$

i.e., containing an additional weight parameter $\epsilon$. The best overall isotopy in this family was (rather loosely) considered and $L_{\epsilon=1 / 2}\left(=L_{\mathrm{OPs}}\right)$ was found optimal. However, it is obvious that the eigenvalue spectrum for the Laplacian matrix $L_{\mathrm{OPs}}$ is $-4 \leqslant \lambda_{j} \leqslant 0$, meaning (see the reasoning in the previous subsection) that the stability region for the CDS scheme in the $A-D$ plane will shrink considerably.

There are two options to deal with this issue. Since all discrete Laplacians carry a prefactor $1 / \Delta x^{2}$ (set to unity by the implicit choice $\Delta x=\Delta x_{\mathrm{OP}}=1$ by Oono and Puri), the first option is to select $\Delta x=\sqrt{\lambda_{L, \min } / \lambda_{\mathrm{OP}, \min }}$ instead, with $\lambda_{\text {min }}$ the lowest eigenvalue of the considered $L$. This choice would lead to a method that employs an actual discrete Laplacian and carries the same stability region as the original scheme. For $L_{\mathrm{OPs}}$, we would thus find $\Delta x^{2}=3 \Delta x_{\mathrm{OP}}^{2}=3$, leading to a scaling of $L$ by a factor $1 / 3$. Indeed, in a 3D CDS study of Shinozaki and Oono [11], the parameter $D$ in the CahnHilliard-Cook model and $D_{\mathrm{CDS}}$, the equivalent parameter in CDS, were said to be "roughly" related as $D=s D_{\mathrm{CDS}}$, from looking at the small-|k| behavior, with $s=1 / 3$ (2D) and $s=$ $11 / 40$ (3D). We find that these factors are exact (see Sec. V on 3D discrete Laplacians) and that they originate from the factor needed for optimal $-\mathbf{k}^{2}$ scaling or, equivalent, from rescaling the eigenvalue spectrum of the discrete Laplacian to that of the equivalent averaging operator. Moreover, we find that the same relation holds between $M$ and $M_{\mathrm{CDS}}$. The second, alternative, option is to introduce a time increment $\Delta t \leqslant 1$, leading to the update scheme

$$
\vec{\psi}_{t+1}=\vec{\psi}_{t}+\Delta t L\left[(1-A) \vec{\psi}_{t}+\frac{A}{3} \Phi^{2} \vec{\psi}_{t}-D L \vec{\psi}_{t}\right] .
$$

A proper choice, providing a stability region in parameter space that is similar to that of the original scheme, is $\Delta t=$ $\left(\lambda_{\mathrm{OP}, \min } / \lambda_{L, \min }\right)^{2}=1 / \Delta x^{4}$. For $L_{\mathrm{OPs}}$, we would therefore find $\Delta t=1 / 9$. We conclude that both options are related through the diffusion constant $D$, since it is the proportionality constant for the distance over which a field diffuses over a given time interval. In the remainder, we will discuss the best choice given these two options.

It is not completely surprising that the second option, the introduction of a time increment, embeds CDS into the standard continuum phase field framework. In fact, expression (21) is equal to the Euler-discretized Cahn-Hilliard-Cook equation (5), provided that the (yet unspecified) matrix $L$ is a finite-difference Laplacian instead of an averaging operation. Moreover, since we recover (10) for $\Delta t=1$, the original CDS can be seen as a special case of the Euler-discretized Cahn-Hilliard-Cook equation, i.e., for $L=L_{\mathrm{OP}}$, meaning that it is this specific choice that makes CDS such an efficient model. This insight gives us the tools to replace the original averaging operation by a finite-difference Laplacian of choice. Meanwhile, for any such $L$, its minimal eigenvalue, relative to the reference $L_{\mathrm{OP}}$, gives us a time step $\Delta t$ for which the CDSbased stability region in the $A-D$ plane is conserved. We can use this information to make an inspired choice of $L$ by weighing the advantage of a large stable time step against the properties of discrete Laplacian, like proper scaling and isotropy.

Discrete Laplacians with good isotropy and scaling behavior are of general significance for grid-based simulation methodology. Most derivations concentrate on the isotropy of the discrete Laplacian, which is a necessity for avoiding gridrelated artifacts. Proper structure factors in field methods for block copolymers as well as correct hydrodynamics behavior in lattice-gas and lattice-Boltzmann (LB) simulations rely on proper isotropy of the discretized Laplacian. Proper scaling behavior, especially for smaller wave vectors, is important for the correct emergence and dynamics of larger scale structure. Computational efficiency favors a choice for the most compact computational cell or stencil, as a restriction to neighboring points in the evaluation of an operator (one stride) is efficient compared to methods that consider also point further away (more strides). Several grid-based discrete Laplacians have been proposed in the literature, primarily in the context of LB, 
like the early ones of Junk and Van der Sman [16,17]. Here, we will consider the most frequent choices and discuss their properties in detail.

\section{THE DISCRETE LAPLACIAN IN TWO DIMENSIONS}

The scaling behavior and isotropy of the discrete Laplacians considered in this paper are best assessed in Fourier space. On a grid, cells labeled by $\mathbf{n}=n_{x} \mathbf{i}+n_{y} \mathbf{j}$, with $n_{x}\left(n_{y}\right)=$ $0, \ldots, L_{x}\left(L_{y}\right)$, give rise to a position $\mathbf{r}=\Delta x \cdot \mathbf{n}$, whereas in reciprocal Fourier space $\mathbf{k}=\left(k_{x}, k_{y}\right)=2 \pi / \Delta x\left(n_{x} / L_{x}, n_{y} / L_{y}\right)$, since $\Delta k \Delta x=2 \pi$. The Fourier domain counterpart of the rescaled Laplacian of Oono and Puri (19) reads

$$
\begin{aligned}
L(\mathbf{k})_{\mathrm{OPs}}= & \frac{1}{2(\Delta x)^{2}}\left\{2 \cos \left(k_{x} \Delta x\right)+2 \cos \left(k_{y} \Delta x\right)\right. \\
& \left.+\cos \left[\left(k_{x}+k_{y}\right) \Delta x\right]+\cos \left[\left(k_{x}-k_{y}\right) \Delta x\right]-6\right\},
\end{aligned}
$$

where the prefactor is determined from Taylor expansions of the function around $\mathbf{n}$ in normal space. Expanding $\cos (x)$ around $x=0$ provides

$$
\begin{aligned}
L(\mathbf{k})_{\mathrm{OPs}}= & -\left(k_{x}^{2}+k_{y}^{2}\right)+\frac{\Delta x^{2}}{12}\left(k_{x}^{2}+k_{y}^{2}\right)^{2} \\
& +\frac{\Delta x^{2}}{12} k_{x}^{2} k_{y}^{2}+O\left(k^{6}\right),
\end{aligned}
$$

which shows that this discrete Laplacian is only isotropic up to second order in $k$. As mentioned before, the eigenvalue spectrum of the matrix $L_{\mathrm{OPs}}$ is $-4 \leqslant \lambda_{j} \leqslant 0$. Other considered one-stride stencils, the five-point star Laplacian of Rogers et al. [20] shares the property of being isotropic up to second order. Only a Laplacian defined on a triangular lattice was identified to be isotropic up to fourth order [18].

The question arises if we can obtain a more isotropic discrete Laplacian on a standard cubic grid. Starting from the general compact Laplacian

$$
L(\mathbf{n})_{2 \mathrm{D}}=c_{1} \sum_{i=1}^{4} f_{i}^{(1)}+c_{2} \sum_{i=1}^{4} f_{i}^{(2)}+c_{0} f^{(0)},
$$

with $c_{j}(j \in\{0,1,2\})$ the weight corresponding to a vector class labeled by $j$, we can derive a general expression in the Fourier domain,

$$
\begin{aligned}
L(\mathbf{k})_{2 D}= & a_{0}-a_{2}\left(k_{x}^{2}+k_{y}^{2}\right)+\frac{a_{4} \Delta x^{2}}{12}\left(k_{x}^{2}+k_{y}^{2}\right)^{2} \\
& +\frac{a_{42} \Delta x^{2}}{12} k_{x}^{2} k_{y}^{2}+O\left(k_{\alpha}^{6}\right)
\end{aligned}
$$

with

$$
\begin{aligned}
a_{0} & =c_{0}+4 c_{1}+4 c_{2} \\
a_{2} & =c_{1}+2 c_{2} \\
a_{4} & =c_{1}+2 c_{2}=a_{2} \\
a_{42} & =8 c_{2}-2 c_{1} .
\end{aligned}
$$

The weights $c_{i}$ should be chosen to satisfy the necessary conditions $a_{0}=0$ and $a_{2}=1$. In particular, weights $\left\{c_{i}\right\}$ are required to be positive for reasons of numerical accuracy.
We see that isotropy up to fourth order in $k$ is obtained by an additional condition $a_{42}=0$. The resulting $3 \times 3$ matrix equation can be solved to yield a unique solution $\left(c_{0}, c_{1}, c_{2}\right)=$ $(-20 / 6,4 / 6,1 / 6)$ and we thus find

$$
\begin{aligned}
L(\mathbf{r}) & =\frac{1}{6}\left(4 \sum_{i=1}^{4} f_{i}^{(1)}+\sum_{i=1}^{4} f_{i}^{(2)}-20 f^{(0)}\right) \\
& =L(\mathbf{r})_{D 2 Q 9},
\end{aligned}
$$

which is the 2D Laplacian from the familiar $D n Q m$ family in lattice hydrodynamics [22]. Expanding up to order eight in $k$ provides

$$
\begin{aligned}
L(\mathbf{k})_{D 2 Q 9}= & -\mathbf{k}^{2}+\frac{\Delta x^{2}}{12} \mathbf{k}^{4}-\frac{a_{6} \Delta x^{4}}{360} \mathbf{k}^{6} \\
& -\frac{a_{64} \Delta x^{4}}{360}\left(k_{x}^{4} k_{y}^{2}+k_{x}^{2} k_{y}^{4}\right)+O\left(k_{\alpha}^{8}\right),
\end{aligned}
$$

with $a_{6}=1$ and $a_{64}=24 c_{2}-3 c_{1}=2$, showing that the discrete Laplacian is indeed isotropic only up to order 4 . The eigenvalue spectrum of the matrix $L_{D 2 Q 9}$ is $-5.333 \leqslant \lambda_{j} \leqslant 0$. Consequently, $\Delta x^{2}=4 \Delta x_{\mathrm{OP}}^{2}$ and $\Delta t=1 / 16$.

To compare the properties of these discrete Laplacians on the relevant $\mathbf{k}$ range, we considered the scaling behavior (along the $k_{x}$ direction) and a measure of the isotropy for the two 2D discrete Laplacians, see Fig. 3. Isotropy is considered, after introducing cylindrical coordinates $(r, \phi)$ in reciprocal $\mathbf{k}$ space, by plotting

$$
d(r)=\max _{\phi} L(r, \phi)-\min _{\phi} L(r, \phi)
$$

or the difference between the maximum and minimum value of $L(\mathbf{k})$ for (constant) radius $r$. From Fig. 3, it is clear that the scaling behavior along the $k_{x}$ direction is equivalent for the two considered discrete Laplacians. The earlier determined isotropy up to fourth order of the $D 2 Q 9$ stencil is reflected in $d(r)=0$ up to $r \approx \pi / 2$ (all values in Fourier space are in units of $1 / \Delta x)$. However, for larger $r$, the stencil becomes rather anisotropic. The scaled Oono-Puri stencil is less isotropic than the $D 2 Q 9$ stencil for $1.5<r<2.5$ but more isotropic for $r>2.5$. Depending on the objective, one could conclude that the isotropy of $L_{\mathrm{OPs}}$ is even better than that of $L_{D 2 Q 9}$, which is a surprise. We note, however, that the anisotropy for large wave vectors of $L_{D 2 Q 9}$ will usually not show up in simulations of phase separation, where the important structure is associated with smaller wave vectors, whereas $L_{\mathrm{OPs}}$ may be slightly anisotropic in this range.

In addition to data for $L_{\mathrm{OPs}}$ and $L_{D 2 Q 9}$, we have also plotted data for another stencil, denoted by a 13-point star, which is isotropic up to sixth order in $k$. In particular, we considered a discrete Laplacian of the form

$$
L(\mathbf{n})_{13 p}=c_{1} \sum_{i=1}^{4} f_{i}^{(1)}+c_{2} \sum_{i=1}^{4} f_{i}^{(2)}+c_{4} \sum_{i=1}^{4} f_{i}^{(4)}+c_{0} f^{(0)},
$$

where we have added $N_{4}=4$ vectors of length $\left\|\mathbf{n}_{i}^{2}\right\|=\sqrt{4}=$ 2 , i.e., the next-next-neighbors along the two Cartesian directions, and used the expansion in Fourier space to determine 

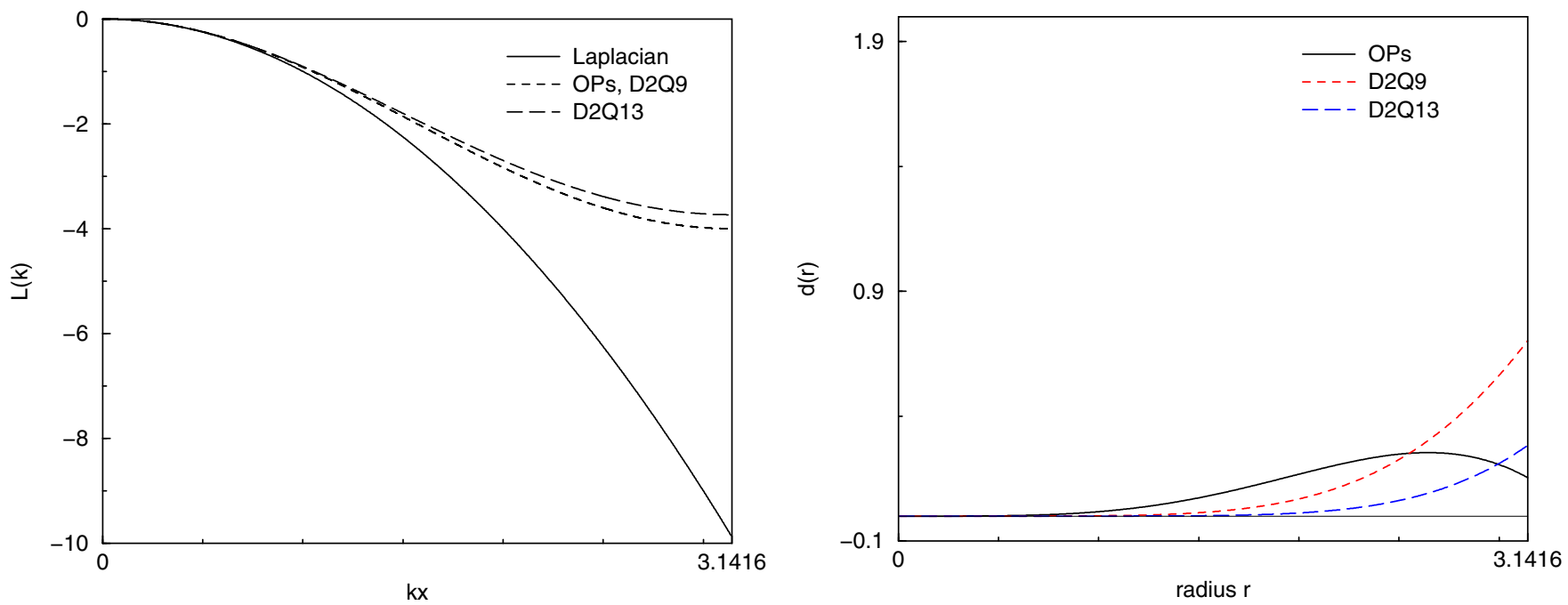

FIG. 3. (Color online) Left: Comparison of $-\mathbf{k}^{2}$ (solid line) and the considered 2D discrete Laplacians along the $k_{x} / \Delta x \in[0, \pi]$ axis. Right: The value of a measure of the isotropy $d(r)$ for the actual Laplacian (thin base line) and the considered discrete Laplacians, $L_{\mathrm{OPs}}$ (solid black line), $L_{D 2 Q 9}$ (red dashed line), and $L_{D 2 Q 13}$ (blue long-dashed line), with $r$ the radius in units of $1 / \Delta x$.

optimal $c_{i}$. The prefactors up to eighth order are now given by

$$
\begin{aligned}
a_{0} & =c_{0}+4 c_{1}+4 c_{2}+4 c_{4} \\
a_{2} & =c_{1}+2 c_{2}+4 c_{4} \\
a_{4} & =c_{1}+2 c_{2}+16 c_{4}=a_{2}+12 c_{4} \\
a_{42} & =8 c_{2}-2 c_{1}-32 c_{4} \\
a_{6} & =c_{1}+2 c_{2}+64 c_{4}=a_{4}+48 c_{4} \\
a_{64} & =24 c_{2}-3 c_{1}-192 c_{4} .
\end{aligned}
$$

Since we have now four unknowns, we can use four conditions $a_{0}=a_{42}=a_{64}=0$ and $a_{2}=1$ to obtain an expression that is isotropic up to order six. The unique solution is $\left(c_{0}, c_{1}, c_{2}, c_{4}\right)=$ $(-180 / 60,32 / 60,12 / 60,1 / 60)$ and the scaling behavior and isotropy measure $d$ for the resulting $D 2 Q 13$ stencil are also shown in Fig. 3. Comparing the isotropy measure for all discrete Laplacians, it is clear that $L_{D 2 Q 13}$ is the most isotropic discrete 2D Laplacian. However, this improved isotropy comes with a penalty. If we focus on the scaling behavior, the two 9-point stencils clearly perform better than the 13-point stencil for larger $|\mathbf{k}|$. Since $c_{4}>0$, this can also be seen from

$$
L_{D 2 Q 9}(\mathbf{k})-L_{D 2 Q 13}(\mathbf{k})=-\frac{12 c_{4} \Delta x^{2}}{12} \mathbf{k}^{4}+O\left(k_{\alpha}^{6}\right) .
$$

We note that this is a standard property for larger stencils, i.e., more than 9 points in $2 \mathrm{D}$ or 27 points in $3 \mathrm{D}$, if the weights $c_{i} \geqslant 0$, as is required for reasons of numerical accuracy.

We thus conclude that the scaling behavior provides a good argument to concentrate on the most compact stencils in two and three dimensions, besides efficiency. In general, the scaling behavior of the discrete Laplacian deviates considerably from $-\mathbf{k}^{2}$ for larger $|\mathbf{k}|$ and cannot be improved upon. This is a direct consequence of approximating this quadratic function by a sum of cosines with positive coefficients and the requirement of proper scaling in the vicinity to the origin.

\section{NUMERICAL EVALUATION IN TWO DIMENSIONS}

To test our predictions and to study the options for scaling, we performed 2D cell dynamics simulations with the map $f(\psi)=A \psi-(A / 3) \psi^{3}$ for a $100 \times 100$ lattice. The parameters $A=1.3$ and $D=0.5$ are well within the "safe" or stable region. Since droplets are ideal to challenge the isotropy of the discrete Laplacian, the initial $\vec{\psi}_{0}$ is filled with $\psi_{i}=$ $-0.3 \pm 0.01$, where the random part is taken from a Gaussian distribution. The results of simulation are shown in Figs. 4-6. We note that the range of $\psi$ values, from $\psi=0.86$ (red, minority phase) to -0.83 (blue, majority phase), respectively, is the same. Figure 4 shows the results of $10^{5}$ steps of CDS for different $L$. Apart from the original averaging operator of Oono and Puri [10], $L_{\mathrm{OP}}$, we have considered the discrete Laplacians $L_{\mathrm{OPs}}$ and $L_{D 2 Q 9}$ and scaled the latter two by $1 / \Delta x^{2}$, with $\Delta x^{2}=3$ (OPS) or 4 (D3Q9), respectively. Although $L_{D 2 Q 9}$ was found to be isotropic up to fourth order in $k$, independent of the grid spacing $\Delta x$, it is obvious from (27) that introducing a grid spacing $\Delta x>1$ amplifies the contribution of the higher-order terms in $k$. Although the (relative) isotropy [determined via $d(r)$ ] can be seen to improve, this results in
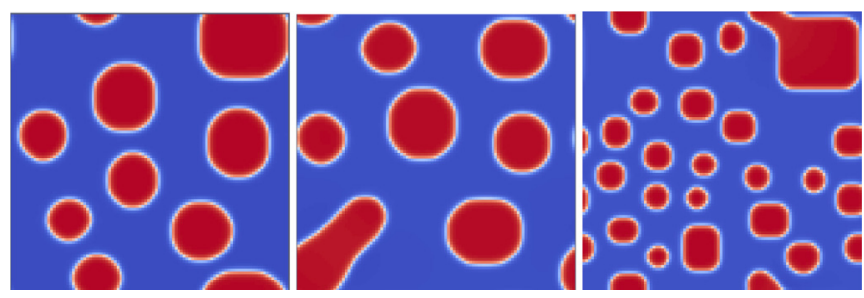

FIG. 4. (Color online) Two-dimensional snapshots for all considered $L$, obtained from CDS with an adaptive grid spacing, after $10^{5}$ steps of CDS. From left to right: $L=L_{\mathrm{OP}}, L=(1 / 3) L_{\mathrm{OPs}}$ $(\Delta x=\sqrt{3})$, and $L=(1 / 4) L_{D 2 Q 9}(\Delta x=2)$. Most clearly visible are the white interfacial regions, which separate domains that are rich of one of the components in the binary blend. 

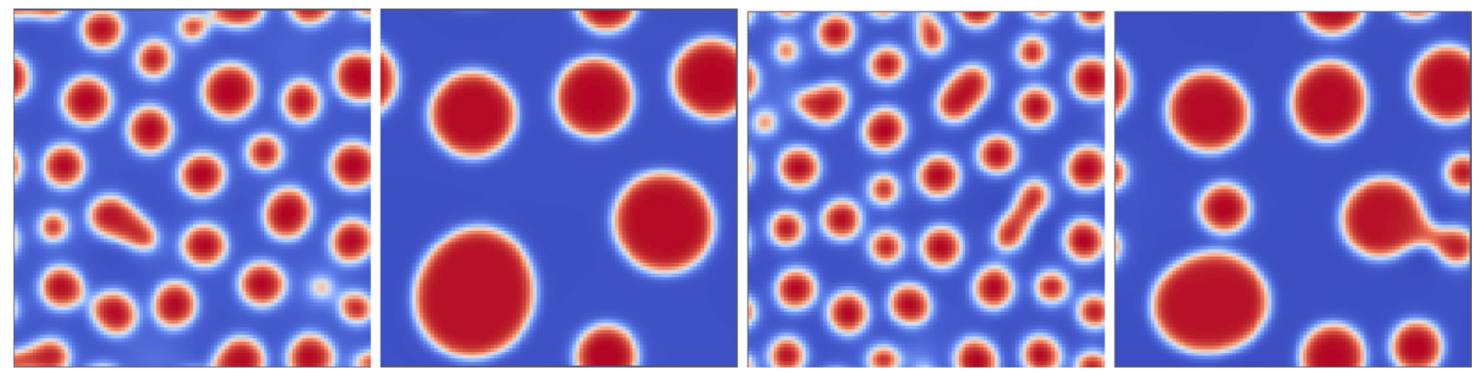

FIG. 5. (Color online) Two-dimensional snapshots obtained by using CDS with an adaptive time step. To simplify a comparison, all stages are counted by the associated number of CDS steps. Panel on the left: $L=L_{\mathrm{OPs}}(\Delta t=1 / 9)$ after $10^{4}$ and $10^{5}$ steps. Two panel on the right: $L=L_{D 2 Q 9}(\Delta t=1 / 16)$ after $10^{4}$ and $10^{5}$ steps. Most clearly visible are the white interfacial regions, which separate domains that are rich of one of the components in the binary blend.

a deteriorating scaling behavior of all considered $L$ on the whole $|\mathbf{k}|$ range and considerable anisotropy effects, even up to the smaller $|\mathbf{k}|$ range where the effect is visible in the snapshots in Fig. 4. Also for the other two operators, $L_{\mathrm{OP}}$ and $L_{\mathrm{OPs}}$, anisotropy is apparent in the somewhat cubic shape of the droplets, although this effect is weaker, as a result of the properties of the particular $L$ and a smaller scaling factor.

If we, instead of scaling by the grid spacing, introduce scaling in the form of a time step $\Delta t$ in CDS, see Fig. 5, as discussed previously, we find spherical droplets for both $L_{\mathrm{OPs}}$ and $L_{D 2 Q 9}$. A difference, in terms of isotropy, between the two discrete Laplacians is not apparent from these results and both simulation results deviate from those obtained using the original $L_{\mathrm{OP}}$ in the interfaces being more diffuse, despite an equivalent degree of demixing. Considering the late stage, after $10^{6}$ steps of CDS, see Fig. 6, the simulation using $L_{\mathrm{OPs}}$ has reached the stage of one perfectly round droplet, as dictated by minimization of the interfacial energy. For $L_{D 2 Q 9}$, instead of one, two large round droplets are found, but we note that, while the number of CDS steps is the same, the time step $\Delta t$ for $L_{D 2 Q 9}$ is smaller and the system can be seen to be at an earlier stage in the coarsening process. Finally, for $L_{\mathrm{OP}}$, the stage after $10^{6}$ steps of CDS is that of a single droplet. However, the droplet is cubic, signaling that simulations based on the original $L$ of Oono and Puri [10] suffer from grid artifacts. Comparing the results for $L_{\mathrm{OP}}$ and $L_{\mathrm{OPs}}$, we find an equivalent structure apart from the droplet shape, and we conclude that improper scaling behavior, in combination with anisotropy in the large- $|\mathbf{k}|$ range, gives rise to grid related artifacts. Overall, we conclude that the introduction of a time step, as discussed above, is the best option for including discrete Laplacians in CDS with proper isotropy.

\section{THE DISCRETE LAPLACIAN IN THREE DIMENSIONS}

In three dimensions, the CDS scheme of Shinozaki and Oono is equivalent to (2), apart from a mobility constant $M$ which was absent in the two-dimensional case [11]. Their 3D averaging operator $\langle\langle *\rangle\rangle-*$ is given by

$$
L(\mathbf{n})_{\text {SO }}=\frac{6}{80} \sum_{i=1}^{6} f_{i}^{(1)}+\frac{3}{80} \sum_{i=1}^{12} f_{i}^{(2)}+\frac{1}{80} \sum_{i=1}^{8} f_{i}^{(3)}-f^{(0)},
$$

where $\Delta x^{2}$ is again assumed to be unity. In this notation, we have $N_{0}=1$ null vector with $\left\|\mathbf{n}^{0}\right\|=0, N_{1}=6$ vectors of length $\left\|\mathbf{n}_{i}^{1}\right\|=1, N_{2}=12$ vectors of length $\left\|\mathbf{n}_{i}^{2}\right\|=\sqrt{2}$ and $N_{3}=8$ vectors of length $\left\|\mathbf{n}_{i}^{3}\right\|=\sqrt{3}$. For the operator defined by (31) we find the eigenvalue spectrum of the associated real and symmetric matrix $L$ as $-1.2 \leqslant \lambda_{j} \leqslant 0$. In the remainder, we will use this operator as a reference for all considered discrete 3D Laplacians, in the spirit that we previously discussed in detail. In particular, we define the factor $\Delta x^{2}$ that can be used to scale the eigenvalue spectrum onto the one associated with of the original (3D) operator $L_{\text {SO }}$, such that the stability conditions will be equivalent. After rescaling the weights in (31) to obtain proper $-\mathbf{k}^{2}$ behavior, i.e.,

$$
L(\mathbf{n})_{\mathrm{SOs}}=\frac{1}{22}\left[6 \sum_{i=1}^{6} f_{i}^{(1)}+3 \sum_{i=1}^{12} f_{i}^{(2)}+\sum_{i=1}^{8} f_{i}^{(3)}-80 f^{(0)}\right],
$$

in units of $1 / \Delta x^{2}$, we find the following expression in the Fourier domain:

$$
\begin{aligned}
L(\mathbf{k})_{\text {SOs }}= & \frac{1}{22 \Delta x^{2}}\left[12 \cos \left(k_{x} \Delta x\right)+12 \cos \left(k_{y} \Delta y\right)+12 \cos \left(k_{z} \Delta z\right)+12 \cos \left(k_{x} \Delta x\right) \cos \left(k_{y} \Delta y\right)\right. \\
& \left.+12 \cos \left(k_{x} \Delta x\right) \cos \left(k_{z} \Delta x\right)+12 \cos \left(k_{y} \Delta x\right) \cos \left(k_{z} \Delta x\right)+8 \cos \left(k_{x} \Delta x\right) \cos \left(k_{y} \Delta x\right) \cos \left(k_{z} \Delta x\right)-80\right] \\
= & -\mathbf{k}^{2}+\Delta x^{2}\left[\frac{\mathbf{k}^{4}}{12}+\frac{2\left(k_{x}^{2} k_{y}^{2}+k_{x}^{2} k_{z}^{2}+k_{y}^{2} k_{z}^{2}\right)}{33}\right]+O\left(k_{\alpha}^{6}\right),
\end{aligned}
$$

showing that this discrete Laplacian $L(\mathbf{k})_{\text {SOs }}$ is isotropic up to second order in $k$. The eigenvalue spectrum of the matrix $L_{\text {SOs }}$ is $-4.3636 \leqslant \lambda_{j} \leqslant 0$. We thus obtain $\Delta x^{2}=40 / 11 \Delta x_{\text {SO }}^{2}$. 

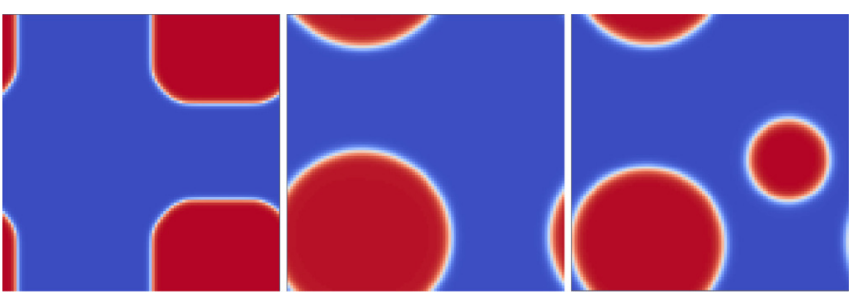

FIG. 6. (Color online) Two-dimensional CDS snapshots for all considered $L$ at late stages. We used an adaptive time step $\Delta t$, given in brackets, but all results are for the same amount of steps $\left(10^{6}\right)$ of CDS. From left to right: $L=L_{\mathrm{OP}}(\Delta t=1.0), L=L_{\mathrm{OPs}}(\Delta t=$ $1 / 9)$, and $L=L_{D 2 Q 9}(\Delta t=1 / 16)$. Most clearly visible are the white interfacial regions, which separate domains that are rich of one of the components in the binary blend.

From the general compact Laplacian in three dimensions

$$
L(\mathbf{n})_{3 D}=c_{1} \sum_{i=1}^{6} f_{i}^{(1)}+c_{2} \sum_{i=1}^{12} f_{i}^{(2)}+c_{3} \sum_{i=1}^{8} f_{i}^{(3)}+c_{0} f^{(0)}
$$

with $c_{j}(j \in\{0,1,2,3\})$ the weight corresponding to a vector class labeled by $j$, we can derive a general expression in the Fourier domain,

$$
\begin{aligned}
L(\mathbf{k})_{3 D}= & a_{0}-a_{2} \mathbf{k}^{2}+\frac{a_{4} \Delta x^{2}}{12} \mathbf{k}^{4} \\
& +\frac{a_{42} \Delta x^{2}}{12}\left(k_{x}^{2} k_{y}^{2}+k_{x}^{2} k_{z}^{2}+k_{y}^{2} k_{z}^{2}\right)+O\left(k_{\alpha}^{6}\right),
\end{aligned}
$$

with

$$
\begin{aligned}
a_{0} & =c_{0}+6 c_{1}+12 c_{2}+8 c_{3} \\
a_{2} & =c_{1}+4 c_{2}+4 c_{3} \\
a_{4} & =a_{2} \\
a_{42} & =4 c_{2}+16 c_{3}-2 c_{1} .
\end{aligned}
$$

The three conditions used for the two-dimensional case, $a_{0}=$ $0, a_{2}=1$, and $a_{42}=0$, are insufficient, since there are now four unknowns. However, by selecting a value for one of the weights, $c_{0}$, we do find a unique solution given by $\left(c_{1}, c_{2}, c_{3}\right)=$ $\left(-c_{0} / 2-5 / 3, c_{0} / 4+7 / 6,-c_{0} / 8-1 / 2\right)$. The additional requirement that all weights $c_{i} \geqslant 0$ sets the value of $c_{0}$ to the range $[-14 / 3,-4]$. Indeed, for $c_{0}$ equal to $-4,-14 / 3$, and $-38 / 9$, we obtain the 3D discrete Laplacians:

$$
\begin{aligned}
L(\mathbf{n})_{D 3 Q 19}= & \frac{1}{6}\left[2 \sum_{i=1}^{6} f_{i}^{(1)}+\sum_{i=1}^{12} f_{i}^{(2)}-24 f^{(0)}\right] \\
L(\mathbf{n})_{D 3 Q 15}= & \frac{1}{12}\left[8 \sum_{i=1}^{6} f_{i}^{(1)}+\sum_{i=1}^{8} f_{i}^{(3)}-56 f^{(0)}\right] \\
L(\mathbf{n})_{D 3 Q 27}= & \frac{1}{36}\left[16 \sum_{i=1}^{6} f_{i}^{(1)}+4 \sum_{i=1}^{12} f_{i}^{(2)}\right. \\
& \left.+\sum_{i=1}^{8} f_{i}^{(3)}-152 f^{(0)}\right],
\end{aligned}
$$

respectively, belonging to the well-known $D n Q m$ class used in lattice hydrodynamics [22]. In addition, for $c_{0}=-128 / 30$ and $c_{0}=-200 / 48$, we obtain the discrete Laplacians of PatraKartunnen and Kumar:

$L(\mathbf{n})_{\mathrm{PK}}=\frac{1}{30}\left[14 \sum_{i=1}^{6} f_{i}^{(1)}+3 \sum_{i=1}^{12} f_{i}^{(2)}+\sum_{i=1}^{8} f_{i}^{(3)}-128 f^{(0)}\right]$

$L(\mathbf{n})_{\mathrm{KU}}=\frac{1}{48}\left[20 \sum_{i=1}^{6} f_{i}^{(1)}+6 \sum_{i=1}^{12} f_{i}^{(2)}+\sum_{i=1}^{8} f_{i}^{(3)}-200 f^{(0)}\right]$.

The first, $L(\mathbf{r})_{\mathrm{PK}}$ [24], has been systematically derived by imposing rotational invariance and isotropy, while $L(\mathbf{r})_{\mathrm{KU}}$ is selected based on the absence of a directional bias [25]. Indeed, all discrete Laplacians in this class are isotropic at fourth order with

$$
L(\mathbf{k})=-\mathbf{k}^{2}+\frac{\mathbf{k}^{4}}{12}+O\left(k_{\alpha}^{6}\right),
$$

but none of them is isotropic up to sixth order. In particular, the sixth-order term is given by

$$
\begin{aligned}
- & \frac{\Delta x^{4}}{360}\left[a_{6} \cdot \mathbf{k}^{6}+a_{64}\left(k_{x}^{4} k_{y}^{2}+k_{x}^{2} k_{y}^{4}+k_{x}^{4} k_{z}^{2}\right.\right. \\
& \left.\left.+k_{x}^{2} k_{z}^{4}+k_{y}^{4} k_{z}^{2}+k_{y}^{2} k_{z}^{4}\right)+a_{62} k_{x}^{2} k_{y}^{2} k_{z}^{2}\right],
\end{aligned}
$$

with $a_{6}=a_{4}=a_{2}, \quad a_{64}=3\left(16 c_{3}+6 c_{2}-c_{1}\right)$, and $a_{62}=$ $6\left(56 c_{3}-4 c_{2}-c_{1}\right)$. We may be tempted to set a fourth condition, $a_{64}=0$, but for that case a unique solution does not exist, as we obtain a matrix with det $=0$, meaning that the fourth condition is linearly dependent on the other three. In particular, filling in the previous solution in terms of $c_{0}$ provides $a_{64}=2$, i.e., independent of $c_{0}$, and $a_{62}=-45 c_{0}-186$. The eigenvalue spectrum of the matrices $L$ associated with these stencils was again determined numerically using a Jacobi method. All spectra take the form $\lambda_{\text {min }} \leqslant \lambda_{j} \leqslant 0$, with $\lambda_{\text {min }}$ as in Table I. The appropriate scaling factor $\Delta x^{2}$ for each of the discrete $3 \mathrm{D}$ Laplacians (with respect to the reference) is added as well.

The isotropy of these discrete Laplacians was recently considered by Thampi et al. [22] in analogy with the analysis in the original study of Shinozaki and Oono [11], via isocontour plots along the $k_{z}=0$ and $k_{z}=\pi$ planes, so we refer to that study for details. However, we note that these isocontour plots only provide qualitative information. Plotting $L(\mathbf{k})$ along the $k_{x}$ axis (see Fig. 7, left) shows proper scaling behavior for small $\mathbf{k}$ vectors, corresponding to structure at a larger scale. We find

TABLE I. The minimal value $\lambda_{\min }$ of the eigenvalue spectrum of the matrix $L \in \mathbb{R}^{n \times n}$, as determined for a $10 \times 10 \times 10$ lattice $(n=1000)$, and the resulting scaling factor $s$ in $\Delta x^{2}=s \Delta x_{\text {SO }}^{2}$. All considered Laplacians that are isotropic up to order four are listed. A good value for the time step $\Delta t$ is $1 / s^{2}$.

\begin{tabular}{lcc}
\hline \hline Name & $\lambda_{\min }$ & $s$ \\
\hline D3Q19 & -5.3333 & $40 / 9$ \\
D3Q15 & -9.3333 & $70 / 9$ \\
D3Q27 & -5.7777 & $130 / 27$ \\
KU & -5.3333 & $40 / 9$ \\
PK & -6.1333 & $46 / 9$ \\
\hline \hline
\end{tabular}



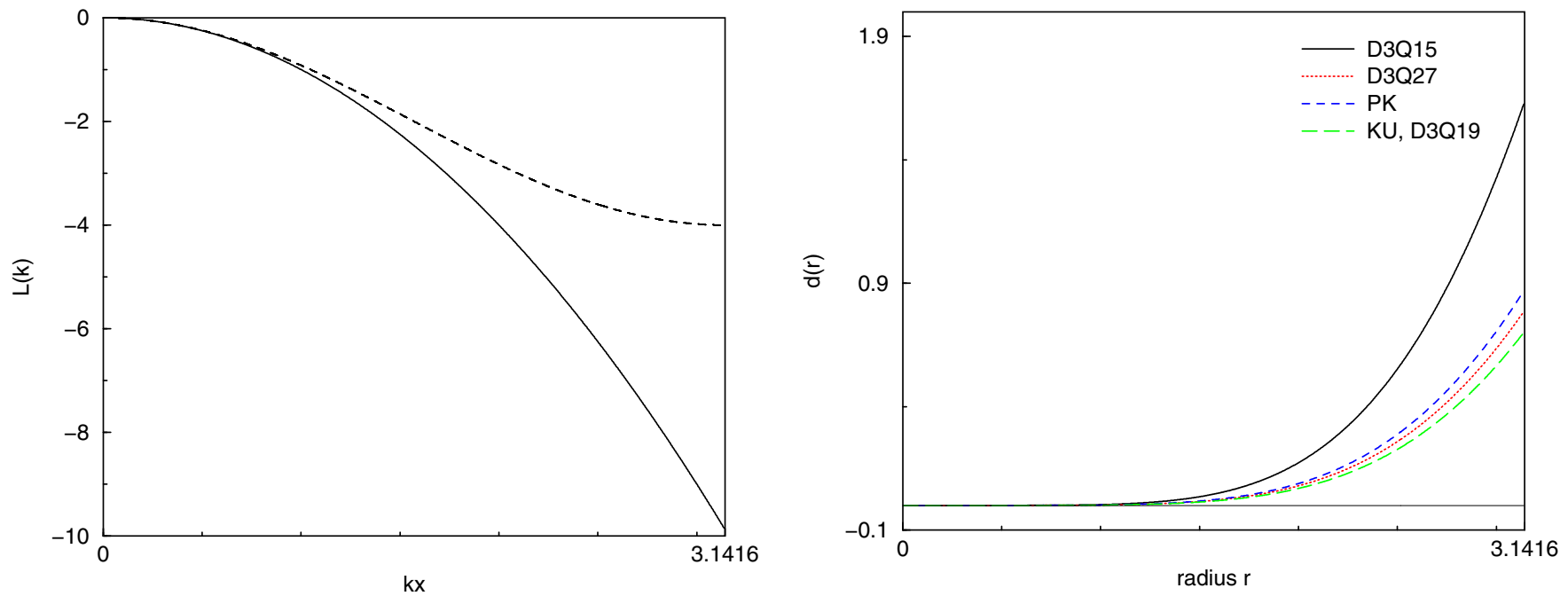

FIG. 7. (Color online) Left: Comparison of $L(\mathbf{k})=-\mathbf{k}^{2}$ (solid line) and all discrete 3D Laplacians considered in this study (they all fall onto a single line, dashed) along the $k_{x} / \Delta x \in[0, \pi]$ axis. Right: The value of a measure of the isotropy $d(r)$ in (39) for the actual Laplacian (thin base line) and the considered discrete Laplacians, $L_{D 3 Q 15}$ (solid black line), $L_{D 3 Q 27}$ (red dotted line), $L_{\mathrm{PK}}$ (blue dashed line), $L_{\mathrm{KU}}$, and $L_{D 3 Q 19}$ (green long dashed line), from the class that are isotropic up to fourth order in $k$, with $r$ the radius in units of $1 / \Delta x$. As the radial plots for $L_{\mathrm{KU}}$ and $L_{D 3 Q 19}$ almost overlap, they have been combined into a single graph.

that, along this direction, all discrete 3D Laplacians considered in this study fall onto a single curve. For larger $\mathbf{k}$ vectors, we again observe that the $-\mathbf{k}^{2}$ scaling of the Laplacian is not very well reproduced for reasons that we explained previously. Isotropy may again be assessed more quantitatively by the measure $d$, after introducing spherical coordinates $(r, \theta, \phi)$ in reciprocal $\mathbf{k}$ space, given by

$$
d(r)=\max _{\theta, \phi} L(r, \theta, \phi)-\min _{\theta, \phi} L(r, \theta, \phi) .
$$

The results for $L_{D 3 Q i}(i=15,19,27), L_{\mathrm{KU}}$, and $L_{\mathrm{PK}}$ are shown in Fig. 7 (right). From the observation that $d(r)=0$ for $r \approx 1$ (in units of $1 / \Delta x$ ), we indeed find proper isotropy for small $r$, in agreement with (37), which is obtained by expanding $\cos (x)$ around the origin. Nevertheless, with increasing $r$, the discrete Laplacians become increasingly anisotropic. Moreover, we conclude that $L_{\mathrm{KU}}$ and $L_{D 3 Q 19}$ are the most isotropic Laplacians on the $0<|\mathbf{k}|<\pi$ range (in units of $1 / \Delta x)$, showing that, although all Laplacians belong to the same class, there are optimal choices.

\section{A. Alternative derivation of a discrete Laplacian}

In dynamic density functional theory (DDFT), we have used a different route to determine a discrete Laplacian with proper scaling behavior and isotropy. Since the derivation was never published in detail, we first briefly review. The discrete Laplace operator $L[f](\mathbf{r})$ is written as

$$
L[f](\mathbf{r})=\sum_{\alpha=1}^{27} \mathcal{D}_{\alpha}^{2}[f](\mathbf{r})
$$

with

$$
\begin{aligned}
& \mathcal{D}_{\alpha}[f](\mathbf{r})=e_{\alpha} \frac{f\left(\mathbf{r}+\frac{\mathbf{r}_{\alpha}}{2}\right)-f\left(\mathbf{r}-\frac{\mathbf{r}_{\alpha}}{2}\right)}{\left\|\mathbf{r}_{\alpha}\right\|} ; \\
& \mathcal{D}_{\alpha}^{2}[f](\mathbf{r})=e_{\alpha}^{2} \frac{f\left(\mathbf{r}+\mathbf{r}_{\alpha}\right)-2 f(\mathbf{r})+f\left(\mathbf{r}-\mathbf{r}_{\alpha}\right)}{\left\|\mathbf{r}_{\alpha}\right\|^{2}},
\end{aligned}
$$

where $\mathbf{r}=\mathbf{n}$ is the center point and $\mathbf{r}_{\alpha}$ (both in units of $\Delta x$ ) are the 27 stencil restricted vectors (in the original notation, these vectors are denoted by $\mathbf{n}_{i}^{j}$ in the previous sections) and $e_{\alpha}$ direction-dependent prefactors that need to be determined. Since this expression is invariant under $\mathbf{r}_{\alpha}=-\mathbf{r}_{\alpha}$, we first reduce the number of grid vectors from 27 to 13 . In the Fourier domain, we find (without the prefactors)

$$
\mathcal{D}_{\alpha}(\mathbf{k})=\frac{2 i}{\left\|\mathbf{r}_{\alpha} \Delta x\right\|} \sin \left(\frac{\mathbf{k r}_{\alpha} \Delta x}{2}\right) f(\mathbf{k}) .
$$

Replacing the prefactors by weights $d_{\alpha}$ and using the condition of isotropy for the discretized differential operator $L[f](\mathbf{r})$, it is easy to see that we can further reduce the number of weights $d_{\alpha}$ from 13 to 3 , one for each of the vector classes denoted by $j$. Our purpose is to determine $\left\{d_{\alpha}\right\}=\left\{d_{1}, d_{2}, d_{3}\right\}$ such that

$$
-\mathbf{k}^{2}-\sum_{\alpha=1}^{\alpha=13} d_{\alpha} \mathcal{D}_{\alpha}(\mathbf{k}) \mathcal{D}_{\alpha}(\mathbf{k})
$$

is minimal and isotropic. Writing it as a (least-squares) minimization problem will result in an overdetermined problem and no solution in the strict sense. Consequently, we determine a linear system of three equations for the three unknowns $\mathbf{d}=\left(d_{1}, d_{2}, d_{3}\right)^{T}$. The conditions that are invoked are such that the isotropy of $L(\mathbf{k})$ is maximal. On the other hand, the long length scaling of the discretized derivatives should be guaranteed identical to the analytical form. The selected three conditions are

$$
\begin{aligned}
\frac{\partial^{2} L}{\partial k_{x}^{2}} & =-2 \\
L(\pi, 0,0) & =L(\pi / \sqrt{2}, \pi / \sqrt{2}, 0) \\
L(\pi, 0,0) & =L(\pi / \sqrt{3}, \pi / \sqrt{3}, \pi / \sqrt{3}) .
\end{aligned}
$$


Solving the resulting equations numerically provides prefactors

$$
\mathbf{d}=\left(\begin{array}{l}
d_{1} \\
d_{2} \\
d_{3}
\end{array}\right)=\left(\begin{array}{l}
0.2947256683 \\
0.2354248727 \\
0.1758184398
\end{array}\right)
$$

and, using the equality

$$
\sin ^{2}(x)=\frac{1}{2}-\frac{1}{2} \cos (2 x),
$$

it is easy to see that we can write this Laplacian in the standard form as

$$
\begin{aligned}
L(\mathbf{n})_{B v V}= & d_{1} \sum_{i=1}^{6} f_{i}^{(1)}+\frac{d_{2}}{2} \sum_{i=1}^{12} f_{i}^{(2)}+\frac{d_{3}}{3} \sum_{i=1}^{8} f_{i}^{(3)} \\
& -\left(6 d_{1}+6 d_{2}+\frac{8}{3} d_{3}\right) f^{(0)} \\
= & c_{1} \sum_{i=1}^{6} f_{i}^{(1)}+c_{2} \sum_{i=1}^{12} f_{i}^{(2)}+c_{3} \sum_{i=1}^{8} f_{i}^{(3)}+c_{0} f^{(0)}
\end{aligned}
$$

with coefficients $c_{1}=d_{1}, c_{2}=d_{2} / 2, c_{3}=d_{3} / 3$, and $c_{0}=$ $-6 d_{1}-6 d_{2}-8 d_{3} / 3$. Filling in these values in the general Fourier expression, we find that $a_{0}=0, a_{2}=a_{4}=d_{1}+2 d_{2}+$ $4 d_{3} / 3=1.0$, and $a_{42} \approx 0.82$, so $L(\mathbf{k})_{B v V}$ is isotropic only up to second order in $k$. Since the coefficients are not in a fractional form, rounding errors may become problematic, but we note that they also play a role during numerical implementation of discrete Laplacians with fractional coefficients. An implementation of the form

$$
\begin{aligned}
L(\mathbf{n})_{B v V}= & d_{1} \sum_{i=1}^{6}\left[f_{i}^{(1)}-f^{(0)}\right]+\frac{d_{2}}{2} \sum_{i=1}^{12}\left(f_{i}^{(2)}-f^{(0)}\right) \\
& +\frac{d_{3}}{3} \sum_{i=1}^{8}\left[f_{i}^{(3)}-f^{(0)}\right]
\end{aligned}
$$

is preferable in terms of numerical accuracy.

The eigenvalue spectra of the matrices $L$ associated with this and other stencils that are isotropic up to second order in $k$ were again determined numerically using a Jacobi method. All spectra take the form $\lambda_{\min } \leqslant \lambda_{j} \leqslant 0$, with $\lambda_{\min }$ as in Table II. The appropriate scaling factor $\Delta x^{2}$ for each of the discrete 3D Laplacians (with respect to the reference) is added as well. Eigenvalue spectra for all discrete 3D Laplacians considered in this section are shown in Fig. 8.

TABLE II. The minimal value $\lambda_{\min }$ of the eigenvalue spectrum of the matrix $L \in \mathbb{R}^{n \times n}$, as determined for a $10 \times 10 \times 10$ lattice $(n=1000)$, and the resulting scaling factor $s$ in $\Delta x^{2}=s \Delta x_{\text {SO }}^{2}$. All considered Laplacians that are isotropic up to order two are listed. A good value for the time step $\Delta t$ is $1 / s^{2}$.

\begin{tabular}{lcc}
\hline \hline Name & $\lambda_{\text {min }}$ & $s$ \\
\hline BvV & -4.4744 & 3.73 \\
CD & -12.0 & 10 \\
EW & -4.0 & $10 / 3$ \\
SOs & -4.3636 & $40 / 11$ \\
\hline \hline
\end{tabular}

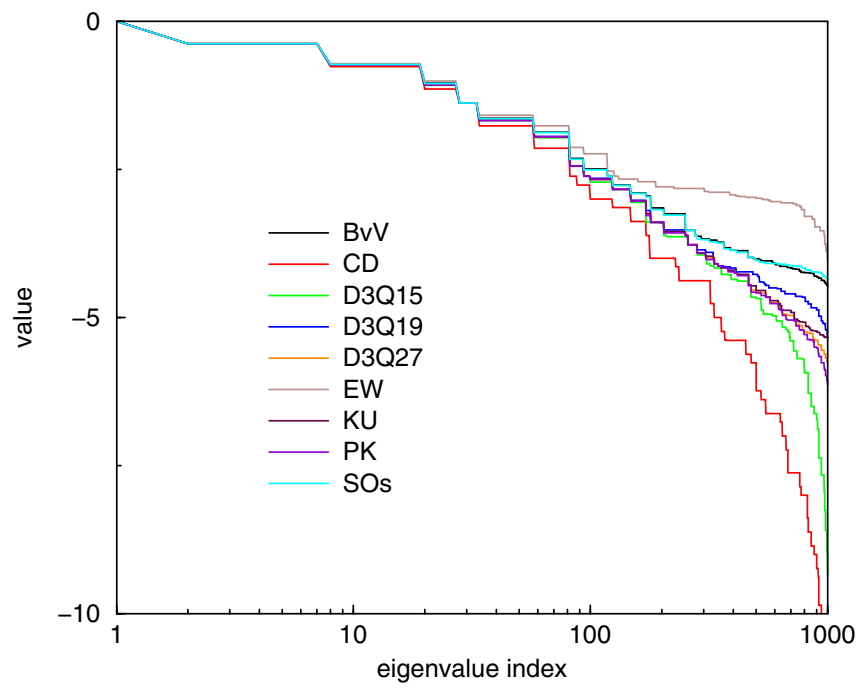

FIG. 8. (Color online) Numerically determined values of the eigenvalue $\lambda_{j}$ of all 3D discrete Laplacians for a $10 \times 10 \times 10$ lattice. Lines are drawn for better visibility. The minimal eigenvalues provided in Tables I and II can be used to distinguish between the different discrete Laplacians in a black-and-white plot.

The isotropy of the discrete 3D Laplacian $L(\mathbf{k})_{B v V}$ is considered in Fig. 9, via the measure $d(r)$. For completeness, also the isotropy measure $d(r)$ for other discrete 3D Laplacians that are only isotropic up to second order, i.e., the simplest central difference (CD), the equally weighted (EW) that is popular in lattice Boltzmann, and the scaled Shinozaki-Oono (SOs) discrete Laplacian, are included. Overall, the less common $L_{B v V}$ and the usual $L_{\mathrm{SOS}}$ stencil are only slightly anisotropic on the complete $|\mathbf{k}|$ range, whereas the $L_{B v V}$ performs slightly better for large $r$ (at the expense of slightly

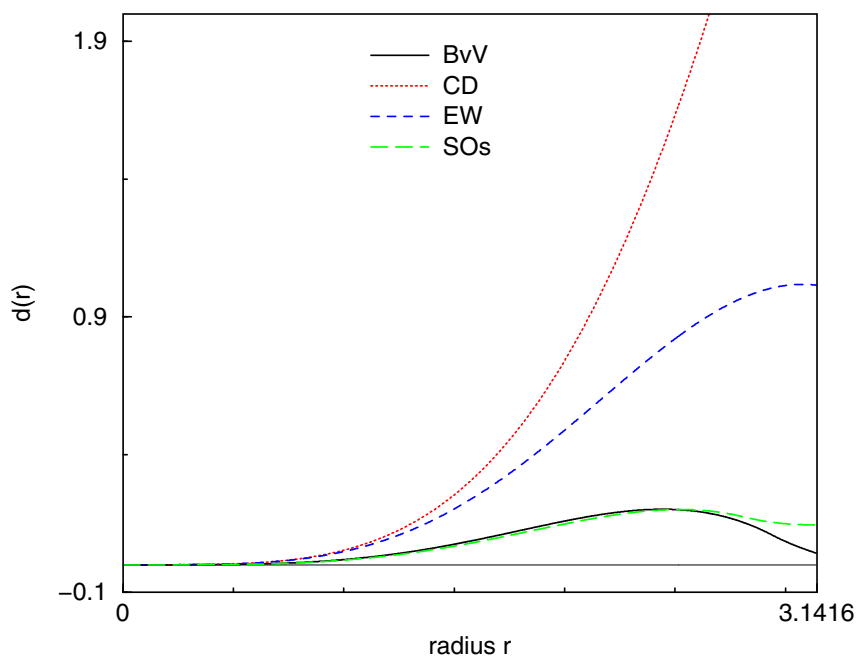

FIG. 9. (Color online) The value of a measure of the isotropy $d(r)$ in (39) for the actual Laplacian (thin base line), $L_{B v V}$ (solid black line), and $L_{\mathrm{SO}}$ (green long dashed line), with $r$ the radius in units of $1 / \Delta x$. The values for other discrete Laplacians that are isotropic up to second order in $k$, the central difference [CD with $\left(c_{0}, c_{1}, c_{2}, c_{3}\right)=$ $(1,0,0,-6)$, red dotted line], and the equally weighted stencil used in lattice Boltzmann [EW with $\left(c_{0}, c_{1}, c_{2}, c_{3}\right)=(1 / 9,1 / 9,1 / 9,-26 / 9)$, blue dashed line], are shown as a reference. 
performing less than $L_{\text {SOs }}$ for smaller $r$ ) due to the specific conditions for which it was derived. The $L_{D 3 Q 19}$ stencil seems the best choice of all considered discrete Laplacians. Apart from being efficient to implement (only 18 directions are considered), it is isotropic in the low $|\mathbf{k}|$ range, up to $|\mathbf{k}| \approx \pi / 2$, and the least anisotropic for larger $|\mathbf{k}|$ values of all considered Laplacians in the class that it belong to. Nevertheless, none of the considered discrete Laplacians is isotropic on the whole range. We thus conclude again that the choice of a discrete 3D Laplacian depends on the specific requirements of the modeler.

\section{CDS FOR OTHER FREE ENERGIES}

So far, we have concentrated on the original model for binary systems of Oono and Puri, but also more complicated systems have been considered, such as block copolymers $[26,27]$ and phase separation with chemical reactions [5,28]. Focusing on $A B$ diblock copolymers, where the order parameter is defined by $\psi=\phi_{A}-\phi_{B}+(1-2 f)$, with $\phi_{I}$ the local volume fraction $I$ monomers $(I \in\{A, B\})$ and $f$ the volume fraction of $A$ monomers in the diblock, the free-energy functional $F[\psi(\mathbf{r}, t)]$ can be written as a sum of short- and long-range interactions [6,27]

$$
\begin{aligned}
\frac{F[\psi]}{k_{B} T}= & \int d \mathbf{r}\left\{H[\psi(\mathbf{r})]+\frac{D}{2}|\nabla \psi(\mathbf{r})|^{2}\right\} \\
& +\frac{B}{2} \int d \mathbf{r} \int d \mathbf{r}^{\prime} G\left(\mathbf{r}-\mathbf{r}^{\prime}\right) \psi(\mathbf{r}) \psi\left(\mathbf{r}^{\prime}\right),
\end{aligned}
$$

with $G$ the Green's function for the Laplace equation, i.e., $\nabla^{2} G\left(\mathbf{r}-\mathbf{r}^{\prime}\right)=-\delta\left(\mathbf{r}-\mathbf{r}^{\prime}\right), D$ a positive constant that plays the role of a diffusion coefficient, $B$ a parameter that introduces a chain-length dependence of the free energy, and

$$
H[\psi]=\left[-\frac{\tau}{2}+\frac{A}{2}(1-2 f)^{2}\right] \psi^{2}+\frac{v}{3}(1-2 f) \psi^{3}+\frac{u}{4} \psi^{4},
$$

where $\tau$ is related to the temperature and $A, v$, and $u$ are phenomenological constants. For completeness, we note that we have adapted our notation to that of earlier publications and that parameters have been reused. The discrete CDS model for the evolution of $\psi(\mathbf{n}, t)$ at time $t$ and cell $\mathbf{n}=\left(n_{x}, n_{y}, n_{z}\right)$ becomes

$$
\begin{aligned}
\psi(\mathbf{n}, t+1)= & \psi(\mathbf{n}, t)-\{\langle\Gamma[\psi(\mathbf{n}, t)]\rangle\rangle \\
& -\Gamma[\psi(\mathbf{n}, t)]+B \psi(\mathbf{n}, t)\},
\end{aligned}
$$

where noise could be added according to the fluctuation-dissipation relation, and

$$
\Gamma[\psi(\mathbf{n}, t)]=g(\psi(\mathbf{n}, t))-\psi(\mathbf{n}, t)+D[\langle\psi(\mathbf{n}, t)\rangle-\psi(\mathbf{n}, t)],
$$

and the so-called (nonlinear) map is given by

$$
g(\psi)=\left[1+\tau-A(1-2 f)^{2}\right] \psi-v(1-2 f) \psi^{2}-u \psi^{3} .
$$

Rewriting the CDS equations in a vector-matrix notation provides

$$
\begin{aligned}
\vec{\psi}_{t+1}= & (1-B) \vec{\psi}_{t}+L\left(\left[A(1-2 f)^{2}-\tau\right] \vec{\psi}_{t}\right. \\
& \left.+v(1-2 f) \Phi \vec{\psi}_{t}+u \Phi^{2} \vec{\psi}_{t}-D L \vec{\psi}_{t}\right) .
\end{aligned}
$$

We limit ourselves to a symmetric diblock $(f=0.5)$ and usual parameter values and leave the other cases for the reader. The map is given by $g(\psi)=(1+\tau) \psi-0.5 \psi^{3}$ for the usual values $u=0.5, v=1.5, D=0.5, B=0.02$, and $A=1.5$ [27], and the CDS equation becomes

$$
\vec{\psi}_{t+1}=0.98 \vec{\psi}_{t}+L\left(-\tau \vec{\psi}_{t}+0.5 \Phi^{2} \vec{\psi}_{t}-0.5 L \vec{\psi}_{t}\right),
$$

with the parameter $\tau$ setting the effective temperature. Writing $\vec{\psi}_{t}=\sum_{j=1}^{n} a_{j} \vec{\psi}^{j}$ and $\vec{\psi}_{t}^{\prime}=\Phi^{2} \vec{\psi}_{t}=\sum_{j=1}^{n} b_{j} \vec{\psi}^{j}$ as before, we find

$$
\vec{\psi}_{t+1}=\sum_{j=1}^{n} a_{j}\left[0.98+\lambda_{j}\left(-\tau+0.5 \frac{b_{j}}{a_{j}}\right)-0.5 \lambda_{j}^{2}\right] \vec{\psi}^{j} .
$$

Instead of the usual $\psi_{i}=g\left(\psi_{i}\right)=(1+\tau) \psi_{i}-1 / 2 \psi_{i}^{3}$, we consider $(1+0.2) \psi_{i}=g\left(\psi_{i}\right)$ and obtain $\psi_{i}=0$ or $\psi_{i}= \pm \sqrt{2 \tau-0.4}(\tau>0.2)$. Introducing the same vectors as before, we can write the update as

$$
\begin{aligned}
\vec{\psi}_{t+1} & =\sum_{j=1}^{n}\left[\left(a_{j}-c_{j}\right) p_{j}+c_{j} p_{j}^{\prime}\right] \vec{\psi}^{j} \\
& =\vec{\psi}_{t+1}^{r}+\sum_{j=1}^{n}\left(a_{j}-c_{j}\right) p_{j} \vec{\psi}^{j}
\end{aligned}
$$

with $p_{j}^{\prime}=\left(0.98-0.2 \lambda_{j}-0.5 \lambda_{j}^{2}\right)\left(\left|p_{j}^{\prime}\right| \leqslant 1\right.$ for all eigenvalues of Oono and Puri's $L$ and $p_{j}^{\prime}=1$ only for $\lambda_{j}=-0.2$ ) and

$$
p_{j}=0.98+\lambda_{j}\left(-\tau+\frac{1}{2} \frac{b_{j}-d_{j}}{a_{j}-c_{j}}\right)-0.5 \lambda_{j}^{2} .
$$

We first take $\psi_{i}^{r}=0 \forall i$ as a reference, i.e., $c_{j}=d_{j}=0$. Setting $\psi_{i}=\psi_{i}^{r}+\delta_{i}=\delta_{i}$, with $\delta_{i}$ a small perturbation, we obtain $\psi_{i}^{3}=\left(\psi_{i}^{r}\right)^{3}+\delta_{i}^{3}=\delta_{i}^{3}$. Neglecting the $b_{j} / a_{j}$ term, which is minute, we find that $-1<p_{j}<1$ for $\tau<0.2$, meaning that $\vec{\psi} \rightarrow \vec{\psi}^{r}=\overrightarrow{0}$ (the fully mixed state) starting from a small perturbation. For $\tau \geqslant 0.2, p_{j} \geqslant 1$ in a range around $\lambda_{j}=-\tau$ and the perturbation will be sustained or amplified. Taking $\tau>0.2$ and $\psi_{i}^{r}= \pm \sqrt{2 \tau-0.4}$ as a reference, we find $\psi_{i}^{3} \approx\left(\psi_{i}^{r}\right)^{3}+3 \delta_{i}\left(\psi_{i}^{r}\right)^{2}$ when $\delta_{i}$ is small. Defining $\vec{\delta}=\left(\delta_{0}, \ldots, \delta_{n}\right) \in \mathbb{R}^{n}$, we thus find that $\vec{\psi}_{t}^{\prime}=$ $\left(\vec{\psi}_{t}^{r}\right)^{\prime}+(6 \tau-1.2) \vec{\delta}$ or, with $\vec{\delta}=\sum_{j=1}^{n} \epsilon_{j} \vec{\psi}^{j}, a_{j}=c_{j}+\epsilon_{j}$ and $b_{j}=d_{j}+\epsilon_{j}(6 \tau-1.2)$. Substitution provides

$$
p_{j}=0.98+2(\tau-0.3) \lambda_{j}-0.5 \lambda_{j}^{2} .
$$

We thus find that the perturbation does not amplify if $0.98+2(\tau-0.3) \lambda_{j}-0.5 \lambda_{j}^{2} \geqslant-1$. Using again the eigenvalue spectrum as determined for the operator $L$ of Oono and Puri, this leads to the condition $\tau \leqslant 0.7125$. We assumed the standard $D=1 / 2$, but analysis along the same lines can be applied to determine the stability region for other values of $D$. We note that the considered free energy is only appropriate for weak segregation, which sets a physical limit to the value of $\tau$. Numerically, i.e. performing 50000 steps of CDS on a $100 \times 100$ lattice, we encounter checkerboard patterns for $\tau \geqslant 0.7$ and arithmetic overflow for $\tau \geqslant 1.0$, so we conclude that our analysis is rather accurate. We also note that proper scaling of the selected discrete Laplacian (by $1 / \Delta x^{2}$ given in 
the tables) will retain the stability range associated with the standard ( $L_{\mathrm{OP}}$ or $\left.L_{\mathrm{SO}}\right)$ averaging operators.

\section{CONCLUSION}

We have analytically considered the stability of the cell dynamics scheme (CDS) that was introduced by Oono and Puri in the late 1980s. CDS has become a popular method for modeling phase separation dynamics in a wealth of systems, because it is considerably more efficient than, for instance, discretized TDGL or CHC, due to a much larger stable effective time step in CDS. By acknowledging the grid-restricted nature of the method and writing the CDS evolution equations in matrix-vector notation, we can use the specific properties of the averaging operator $\langle\langle *\rangle\rangle-*$ to rationalize the apparent "puzzling" stability of the CDS scheme. From this analysis, it is clear that the original remarks of the CDS developers, i.e., that this averaging operator is not the discrete Laplacian and that the CDS method should not be analyzed via the TDGL equations, make much sense. However, one can replace the original averaging operator by a discrete representation of the continuum Laplacian via the introduction of a scaling, which conserves the region in parameter space where the CDS scheme is stable. This opens up a route for selecting alternative and more isotropic "averaging operators" (which is now an actual discrete Laplacian) and considerably reduce grid-related artifacts in CDS results. We found that the introduction of a time step in CDS is the best option for this scaling and that a proper time step can be calculated from the eigenvalues of the discrete Laplacian, which leads to a simple relation between CDS and the well-known Cahn-HilliardCook equation. Here we determined the eigenvalue spectra numerically, by a Jacobi method for a number of well and lesser-known discrete Laplacians in two and three dimensions. The results, however, suggest than analytic determination should be possible for these special matrices $L$, which have a zero row sum.
Subsequently, we analyzed the scaling properties and isotropy of several discrete Laplacians on a more quantitative footing than previously considered [22]. Evaluating these properties is of general significance, as they are employed in many field-based methodologies. The necessity of good scaling for low $|\mathbf{k}|$ values compromises the scaling behavior for large $|\mathbf{k}|$, and we believe that the consequence of this constraint is not widely realized. We found that the isotropy of the (normalized) discrete Laplacian of Oono and Puri (2D) and Shinozaki and Oono (3D) is surprisingly good, although the isotropy of a number of familiar Laplacians developed for lattice hydrodynamics is better at low $|\mathbf{k}|$, i.e., where in many cases most of the interesting structure develops. For higher $|\mathbf{k}|$, however, the isotropy of these Laplacians is actually worse than for Laplacians that are considered to be less isotropic from an expansion around the origin. Including more points in the stencils introduces a possibility to improve upon the isotropy for larger $|\mathbf{k}|$, but it is not a solution for this issue. Besides being computationally more demanding, isotropy is seen to be closely linked with scaling behavior, and the latter becomes worse upon including additional points. The $D 3 Q 19$, KU, the normalized discrete operator of Shinozaki and Oono, SOs, and the less familiar $B v V$ discrete Laplacians, where the latter was developed for dynamic density functional theory, are among the best choices in 3D. The real preference will depend on the requirements of the modeler, taking into account the properties discussed here.

\section{ACKNOWLEDGMENT}

The author thanks Andrei Zvelindovsky (University of Lincoln, UK) for reminding him of never-published work on discrete Laplacians. Yoshitsugu Oono (University of Illinois, USA) is gratefully acknowledged for sharing his insights into the philosophy behind CDS and for commenting on an earlier version of the manuscript.
[1] A. J. Bray, Theory of phase-ordering kinetics, Adv. Phys. 43, 357 (1994).

[2] C. Harrison, D. H. Adamson, Z. D. Cheng, J. M. Sebastian, S. Sethuraman, D. A. Huse, R. A. Register, and P. M. Chaikin, Mechanism of ordering in striped patterns, Science 290, 1558 (2000).

[3] S. Puri and N. Parekh, Non-algebraic domain growth in binary alloys with quenched disorder, J. Phys. A: Math. Gen. 25, 4127 (1992).

[4] L. Tsarkova, G. J. A. Sevink, and G. Krausch, Nanopattern evolution in block copolymer films: Experiment, simulations and challenges, in Advances in Polymer Science, edited by A. H. E. Müller and H. W. Schmidt (Springer-Verlag, Berlin, 2010), Vol. 227, p. 33.

[5] S. Puri and H. L. Frisch, Segregation dynamics of binary mixtures with simple chemical reactions, J. Phys. A: Math. Gen. 27, 6027 (1994).

[6] M. Pinna and A. V. Zvelindovsky, Large scale simulation of block copolymers with cell dynamics, Eur. Phys. J. B 85, 210 (2012).
[7] H. Emmerich, Advances of and by phase-field modeling in condensed-matter physics, Adv. Phys. 57, 1 (2008).

[8] Y. Oono and S. Puri, Computationally efficient modeling of ordering of quenched phases, Phys. Rev. Lett. 58, 836 (1987).

[9] Y. Oono, The Nonlinear World: Conceptual Analysis and Phenomenology, Springer Series in Synergetics, (Springer-Verlag, Berlin, 2013).

[10] Y. Oono and S. Puri, Study of phase-separation dynamics by use of cell dynamical systems. 1. Modeling, Phys. Rev. A 38, 434 (1988).

[11] A. Shinozaki and Y. Oono, Spinodal decomposition in 3-space, Phys. Rev. E 48, 2622 (1993).

[12] S. Puri and Y. Oono, Study of phase-separation dynamics by use of cell dynamical systems. 2. Two-dimensional demonstrations, Phys. Rev. A 38, 1542 (1988).

[13] I. W. Hamley, Cell dynamics simulations of block copolymers, Macromol. Theory Simul. 9, 363 (2000).

[14] N. Parekh and S. Puri, A new numerical scheme for the Fischer equation, J. Phys. A: Math. Gen. 23, L1085 (1990). 
[15] G. J. A. Sevink, M. Pinna, K. M. Langner, and A. V. Zvelindovsky, Selective disordering of lamella-forming block copolymers under an electric field, Soft Matter 7, 5161 (2011).

[16] M. Junk, A finite difference interpretation of the lattice Boltzmann method, Numer. Method. Part. Differ. Eq. 17, 383 (2001).

[17] R. G. M. van der Sman, Finite Boltzmann schemes, Comput. Fluids 35, 849 (2006).

[18] P. I. C. Teixeira and B. M. Mulder, Comment on "Study of phase-separation dynamics by use of cell dynamical systems. 1 . Modeling,” Phys. Rev. E 55, 3789 (1997).

[19] M. Cheng and A. D. Rutenberg, Maximally fast coarsening algorithms, Phys. Rev. E 72, 055701 (2005).

[20] T. M. Rogers, K. R. Elder, and R. C. Desai, Numerical study of the late stages of spinodal decomposition, Phys. Rev. B 37, 9638 (1988).

[21] M. Grant, M. S. Miguel, J. Viñals, and J. D. Gunton, Theory for the early stages of phase separation: The long-range-force limit, Phys. Rev. B 31, 3027 (1985).
[22] S. P. Thampi, S. Ansumali, R. Adhikari, and S. Succi, Isotropic discrete Laplacian operators from lattice hydrodynamics, J. Comp. Phys. 234, 1 (2013).

[23] H. Tomita, Preservation of isotropy at the mesoscopic stage of phase-separation processes, Progr. Theor. Phys. 85, 47 (1991).

[24] M. Patra and M. Karttunen, Stencils with isotropic discretization error for differential operators, Numer. Method. Part. Differ. Eq. 22, 936 (2006).

[25] A. Kumar, Isotropic finite-differences, J. Comput. Phys. 201, 109 (2004).

[26] M. Bahiana and Y. Oono, Cell dynamic system approach to block copolymers, Phys. Rev. A 41, 6763 (1990).

[27] S. Y. Qi and Z.-G. Wang, Kinetics of phase transitions in weakly segregated block copolymers: Pseudostable and transient states, Phys. Rev. E 55, 1682 (1997).

[28] S. C. Glotzer, E. A. Di Marzio, and M. Muthukumar, Chemically controlled pattern-formation in phase-separating materials, Nouvo Cimento 16, 1171 (1994). 